\title{
Performance Analysis of a Cognitive Radio Network With a Buffered Relay
}

\author{
Hung Tran, Hans-Jürgen Zepernick, Senior Member, IEEE, Hoc Phan, and Louis Sibomana
}

\begin{abstract}
In this paper, we analyze the packet transmission time in a cognitive cooperative radio network (CCRN) where a secondary transmitter (SU-Tx) sends packets to a secondary receiver (SU-Rx) with the help of a secondary relay (SR). In particular, we assume that the $S U-T x$ and the $S R$ are subject to the joint constraint of the timeout probability of the primary user (PU) and the peak transmit power of the secondary users (SUs). On this basis, we investigate the impact of the transmit power of the PUs and channel mean power on the packet transmission time of the CCRN. Utilizing the concept of timeout, adaptive transmit powerallocation policies for the SU-Tx and SR are considered. More importantly, analytical expressions for the end-to-end throughput, end-to-end packet transmission time, and stable condition for the SR operation are obtained. Our results indicate that the second hop of the considered CCRN is not a bottleneck if the channel mean power of the interference links of the networks is small and if the SR peak transmit power is set to a high value.
\end{abstract}

Index Terms-Cognitive relay networks, device-to-device communications, packet transmission time, power control, spectrum sharing, stable condition, throughput.

\section{INTRODUCTION}

$\mathbf{R}$ ECENTLY, cognitive radio networks (CRNs) have been considered a promising technology to deal with the underutilization of spectrum resources in wireless communications [1]. The key idea behind CRNs is to let the secondary user (SU) exploit the licensed frequency bands, which are readily assigned to the primary users (PUs), without degrading the performance of the PUs. Specifically, three main spectrum access methods have been proposed, i.e., overlay, interweave, and underlay [2]. In the overlay paradigm, the SU can simultaneously access a licensed frequency band with the PUs by using a sophisticated coding technique (e.g., dirty paper coding) to cancel the interference to the PU. In the interweave system, the SU needs to detect the temporarily unused spectrum before accessing the free spectrum and must vacate it when it

Manuscript received February 26, 2013; revised July 3, 2013, October 14, 2013, January 20, 2014, and March 26, 2014; accepted April 17, 2014. Date of publication May 13, 2014; date of current version February 9, 2015. This work was supported in part by Vietnam's National Foundation for Science and Technology Development (NAFOSTED). The review of this paper was coordinated by Prof. C.-X. Wang.

H. Tran is with the Network Systems Section, Department of Information Technology, National Institute of Education Management, Hanoi, Vietnam.

H.-J. Zepernick is with Blekinge Institute of Technology, 37141 Karlskrona, Sweden (e-mail: hjz@bth.se).

H. Phan is with the University of Reading, Berkshire RG6 6AH, U.K.

L. Sibomana is with the Department of Electrical and Electronic Engineering, National University of Rwanda, Butare, Rwanda, and also with the Radio Communications Group, Blekinge Institute of Technology, 37141 Karlskrona, Sweden (e-mail: 1sm@bth.se).

Digital Object Identifier 10.1109/TVT.2014.2323338 is reoccupied by the PU. On the other hand, in the underlay system, the SU is allowed to simultaneously access the licensed frequency band of the PU, as long as the interference caused by the SU to the primary receiver (PU-Rx) is below a given threshold. Hence, the secondary transmitter (SU-Tx) transmit power is often kept at a low level, and a direct communication between the SU-Tx and the secondary receiver (SU-Rx) can be only maintained for a short range. In particular, in [3], subject to different constraints, e.g., peak interference power and an outage probability constraint of the PU-Rx, and peak transmit power of the SU-Tx, the performance for various spectrum sharing systems has been analyzed. In [4], given the peak interference power constraint, the delay performance for point-to-point and point-to-multipoint communications has been investigated by utilizing an M/G/1 queuing model.

To overcome such coverage limitations in underlay CRNs, the concept of cognitive cooperative radio network (CCRN) has been introduced as a promising solution [5]-[21]. It has been shown that the coverage range and link reliability of CRNs can be increased significantly through the help of secondary relays (SRs). More specifically, in [5], Simeone et al. have focused on the cooperation between the SU and PU, where the SU-Tx operates as a relay to support the transmission of the primary network. Given this setting, a power-allocation strategy has been derived for the SU-Tx, and the stable throughput for the secondary network has been analyzed. In [6], Sadek et al. have proposed two protocols for cognitive multiple access by cooperation, for which the maximal throughput region and delay performance have been analyzed. In [17], Lee et al. have investigated the diversity order of a CCRN with decode-andforward (DF) relaying and studied the impact of the distances between the terminals on outage performance. In [19] and [22], the asymptotic outage behavior and the effect of interference from the PU on the outage performance of CCRNs with amplify-and-forward (AF) and DF relaying have been examined. In [15] and [18], the outage performance of a CCRN with a single DF relay over Nakagami- $m$ fading channels has been considered. It has been shown that the outage probability depends on both the fading severity parameters and the number of active PUs that are present near the SU. In the aforementioned works and the references therein, the authors have investigated CCRN scenarios in which the relay operates in half-duplex mode, i.e., the relay cannot receive and transmit packets at the same time. Most recently, in [23], the optimal power allocation and outage performance for a CCRN with full-duplex relaying have been investigated. Potential system deployments that combine cognitive radio and cooperative communications can be found, e.g., in [24]. As for surveys 
on recent advances in demonstrators and trails in commercial systems of CR concepts, respectively, see [25] and [26].

Motivated by all of the above, in this paper, we study the performance of a CCRN with the SR operating in full-duplex mode. More specifically, we consider the case that the SR receives packets from the SU-Tx, decodes, and then forwards them to the SU-Rx over orthogonal channels. To assure the desired performance of the PU, the SU-Tx and SR must control their transmit power to meet both the timeout probability constraint of the PUs and the peak transmit power constraint of the SUs. Given these settings, the performance analysis for the considered system is developed. Main contributions in this paper are summarized as follows.

- Adaptive transmit power control policies for the SU-Tx and SR are proposed.

- Using the timeout concept, the cumulative distribution function (cdf) and probability density function (pdf) for the packet transmission time and timeout probability are derived for the secondary network.

- By employing the GI/G/1 (general independent interarrival time, general service time, and a single server) queueing model, analytical expressions for the end-to-end throughput, end-to-end packet transmission time, and stable condition for the SR operation are derived.

- Our results show that if the peak transmit power of the SR is set to a high value and the channel mean power of the interference links between the secondary and primary networks is low, then the SR $\rightarrow$ SU-Rx link is not a bottleneck.

The remainder of this paper is organized as follows. In Section II, the system model and assumptions for the CCRN are introduced. In Section III, adaptive transmit power policies for the SU-Tx and SR under the joint peak transmit power constraint of the SU and the timeout probability constraint of the PU are analyzed. Accordingly, expressions for the pdf and cdf of packet transmission time, timeout probability, end-to-end transmission time, end-to-end throughput, and stable condition for the SR operation are derived. In Section IV, numerical results and discussions are provided. Finally, conclusions are presented in Section V.

Notation: The cdf and pdf of a random variable (RV) $Y$ are denoted by $F_{Y}(\cdot)$ and $f_{Y}(\cdot)$, respectively. The terms $\operatorname{Pr}\{\cdot\}$ and $\operatorname{Pr}\{\cdot \mid \cdot\}$ denote the probability and conditional probability, respectively. $\mathbb{E}[\cdot]$ and $\mathbf{V a r}[\cdot]$ stand for expectation and variance, respectively. $X^{(a, b)}$ denotes a variable that belongs to the $a$ th network and the $b$ th hop where $a \in\{s, p\}$ and $b \in\{1,2\}$. Acronyms $s$ and $p$ represent the secondary and primary networks, respectively.

\section{System Model of the Considered Cognitive CoOperative Radio NeTwORK}

\section{A. System Model}

Let us consider a dual-hop CCRN, as shown in Fig. 1, in which the SU-Tx has a stream of packets to transmit to the SURx through the help of a full-duplex DF relay. In particular, in the first hop, the communication over the SU-Tx $\rightarrow$ SR link utilizes the licensed spectrum of the PUs in Region I. In the

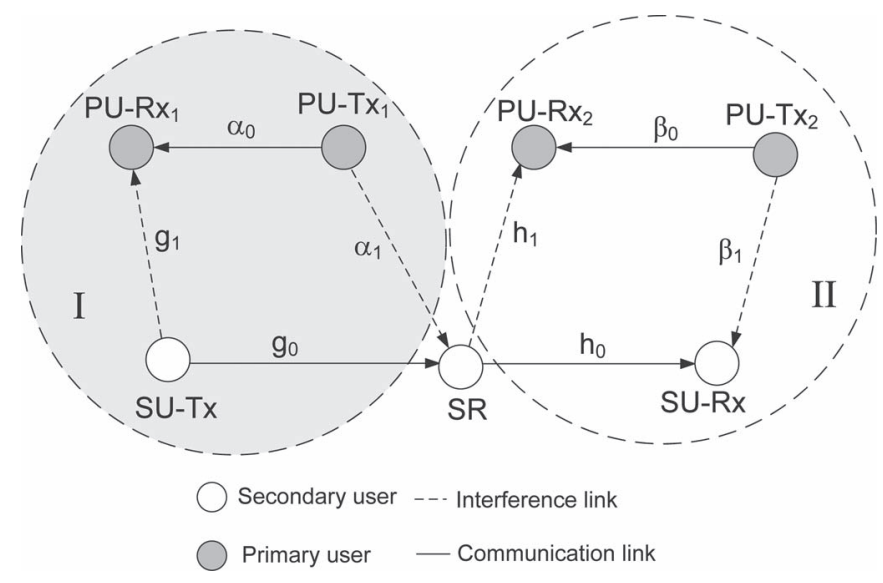

Fig. 1. System model of a CCRN, where the SR receives a packet from the SU-Tx in the first hop, decodes, and forwards it to the SU-Rx in the second hop. The SU-tx $\rightarrow$ SR link utilizes the licensed frequency band of the PUs in Region I, whereas the SR $\rightarrow$ SU-Rx link exploits the licensed frequency band of the PUs in Region II.

second hop, the communication over the $\mathrm{SR} \rightarrow \mathrm{SU}-\mathrm{Rx}$ link uses the licensed frequency band of the PUs in Region II. Frequencies in the regions are assumed orthogonal, and the direct communication between SU-Tx and SU-Rx is not available due to shadowing. Accordingly, the SUs and PUs may cause mutual interference in the same region, but they do not cause interference to users in the other region.

It should be mentioned that the considered scenario is applicable in practice where the SR may act as a base station, whereas the SU-Tx $\rightarrow$ SR and SR $\rightarrow$ SU-Rx links are the uplink and downlink, respectively. Furthermore, this type of system model is an example of a device-to-device communication system, where the primary transmitter (PU-Tx) $\rightarrow$ PU-Rx link is considered uplink or downlink in a cellular network and the SUTx, SR, and SU-Rx are devices that operate in underlay mode [27]-[29]. We also recall that potential system deployments combining $\mathrm{CR}$ and cooperative communications can be found, for example, in [24] and the references therein. Finally, we would like to draw the attention to femtocell deployments where the concept of cooperation and interference avoidance inherent with CCRNs may be applied. A benefit of a femtocell deployment is that service providers can extend service coverage indoors or at the cell edge where access would be limited or unavailable otherwise. Most importantly, a femtocell-based deployment can work with existing mobiles but only requires installation of a new transceiver device or an access point that uses licensed spectrum [30]-[32].

\section{B. Channel Model}

As for the radio links between different users, we assume independent but not necessarily identically distributed (i.n.i.d.) Rayleigh block fading channels. Therefore, the channels are considered constant during the transmission time of one packet but they may change independently to different values thereafter. The channel power gains of the $\mathrm{SU}-\mathrm{Tx} \rightarrow \mathrm{SR}, \mathrm{SR} \rightarrow$ SU-Rx, PU-Tx $x_{1} \rightarrow$ PU-Rx $_{1}$, and PU-Tx $x_{2} \rightarrow$ PU-Rx $_{2}$ communication links are denoted by $g_{0}, h_{0}, \alpha_{0}$, and $\beta_{0}$, respectively. The channel power gains of the $\mathrm{SU}-\mathrm{Tx} \rightarrow \mathrm{PU}^{\mathrm{R} x_{1}}, \mathrm{SR} \rightarrow$ 
PU-Rx 2 , PU-Tx $x_{1} \rightarrow$ SR, and PU-Tx $2 \rightarrow$ SU-Rx interference links are denoted by $g_{1}, h_{1}, \alpha_{1}$, and $\beta_{1}$, respectively. In addition, the channel mean power values of the exponentially distributed RVs $g_{0}, g_{1}, h_{0}, h_{1}, \alpha_{0}, \alpha_{1}, \beta_{0}$, and $\beta_{1}$ are expressed by $\Omega_{g_{0}}, \Omega_{g_{1}}, \Omega_{h_{0}}, \Omega_{h_{1}}, \Omega_{\alpha_{0}}, \Omega_{\alpha_{1}}, \Omega_{\beta_{0}}$, and $\Omega_{\beta_{1}}$, respectively. As the PUs have the highest priority to access their spectrum, they can use an arbitrary power level for the communication without caring about the existence of the SUs. On the other hand, the existence of the SUs should not degrade the performance of the PUs. Hence, the SUs (i.e., SU-Tx and SR) should utilize average channel gains (not instantaneous channel gains) of the SU-Tx $\rightarrow \mathrm{PU}^{-\mathrm{Rx}_{1}}$ and $\mathrm{SR} \rightarrow \mathrm{PU}^{-\mathrm{Rx}_{2}}$ links to control their transmit power. This is due to the fact that a secondary network does usually not collaborate with the primary network and there is no dedicated feedback channel available from the PUs to the SUs. Therefore, the instantaneous channel state information (CSI) of the PUs may be not available at the SUs. However, the SUs can estimate the average channel gains of the PUs by utilizing parameters such as transmission distance, transmit/receive antenna gain, etc., which are considered relatively stable parameters. Furthermore, the average channel gain estimated at the SUs may also save feedback channel resources. In addition, the SU-Tx and SR are assumed to have full CSI of the SU-Tx $\rightarrow$ SR and SR $\rightarrow$ SU-Rx links, respectively. This is reasonable as both SU-Tx and SR are in the same system and should have a dedicated feedback channel [33].

\section{Timeout Probability Constraint of the PU}

Let the PU-Tx $x_{m}, m=1,2$, transmit each packet of length $L_{p}$ bits to the corresponding PU-Rx $x_{m}$. Since the PUs share their licensed spectrum with the SUs, they may be subject to interference caused by the SUs. As such, the performance of the PU is guaranteed if the probability of dropped packets due to timeout is smaller than a predefined threshold. In the following, we will formulate this setting mathematically in terms of a timeout probability constraint.

For the PU-Tx $x_{1} \rightarrow \mathrm{PU}^{\mathrm{Rx}} \mathrm{P}_{1}$ communication, $\mathrm{PU}-\mathrm{Tx}_{1}$ uses transmit power $\mathrm{P}^{(p, 1)}$ to send a packet to the $\mathrm{PU}-\mathrm{Rx}_{1}$. The time taken by the PU-Tx 1 to transmit a packet to the $\mathrm{PU}-\mathrm{Rx}_{1}$ can be formulated as [34]

$$
T^{(p, 1)}=\frac{L_{p}}{B \log _{2}\left(1+\gamma^{(p, 1)}\right)} \triangleq \frac{\tilde{B}_{p}}{\ln \left(1+\gamma^{(p, 1)}\right)}
$$

where $\tilde{B}_{p}=L_{p} \ln (2) / B, B$ is the system bandwidth, and $\gamma^{(p, 1)}$ is the instantaneous signal-to-interference-plus-noise ratio (SINR) at PU-Rx $x_{1}$, which is given by

$$
\gamma^{(p, 1)}=\frac{\mathrm{P}^{(p, 1)} \alpha_{0}}{\mathrm{P}^{(s, 1)} g_{1}+N_{0}}
$$

where $\mathrm{P}^{(s, 1)}$ and $N_{0}$ stand for the transmit power of the SU-Tx and noise power, respectively.

A packet is considered being successfully transmitted if its transmission time $T^{(p, 1)}$, given in (1), is below a predefined timeout threshold $t_{\text {out }}^{p}$. In other words, the probability of un- successful packet reception for $\mathrm{PU}-\mathrm{Rx}_{1}$, known as timeout probability $P_{\text {out }}^{(p, 1)}$, can be formulated as

$$
\begin{aligned}
P_{\text {out }}^{(p, 1)} & =\operatorname{Pr}\left\{T^{(p, 1)} \geq t_{\text {out }}^{p}\right\} \\
& =\operatorname{Pr}\left\{\tilde{B}_{p}\left[\ln \left(1+\frac{\mathrm{P}^{(p, 1)} \alpha_{0}}{\mathrm{P}^{(s, 1)} g_{1}+N_{0}}\right)\right]^{-1} \geq t_{\text {out }}^{p}\right\} .
\end{aligned}
$$

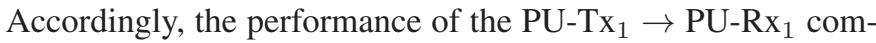
munication in the presence of the $\mathrm{SU}-\mathrm{Tx} \rightarrow \mathrm{SR}$ communication is assured if and only if the timeout probability $P_{\text {out }}^{(p, 1)}$, which is given in (3), satisfies the following condition:

$$
P_{\text {out }}^{(p, 1)} \leq \theta_{\text {th }}^{(p, 1)}
$$

where $\theta_{\mathrm{th}}^{(p, 1)}$ is the timeout probability constraint for the $\mathrm{PU}^{-\mathrm{Rx}_{1}}$.

Similarly, the packet transmission time of the PU-Tx $x_{2} \rightarrow$ PU- $\mathrm{Rx}_{2}$ link is expressed as

$$
T^{(p, 2)}=\frac{L_{p}}{B \log _{2}\left(1+\gamma^{(p, 2)}\right)} \triangleq \frac{\tilde{B}_{p}}{\ln \left(1+\gamma^{(p, 2)}\right)}
$$

where $\gamma^{(p, 2)}$ is the instantaneous SINR at the PU- $\mathrm{Rx}_{2}$ given by

$$
\gamma^{(p, 2)}=\frac{\mathrm{P}^{(p, 2)} \beta_{0}}{\mathrm{P}^{(s, 2)} h_{1}+N_{0}}
$$

where $\mathrm{P}^{(p, 2)}$ and $\mathrm{P}^{(s, 2)}$ are the transmit power of the PU-Tx $\mathrm{x}_{2}$ and $\mathrm{SR}$, respectively. Then, the timeout probability of the $\mathrm{PU}-\mathrm{Rx}_{2}$ in the presence of the $\mathrm{SR} \rightarrow \mathrm{SU}-\mathrm{Rx}$ communication is formulated as

$$
\begin{aligned}
P_{\text {out }}^{(p, 2)} & =\operatorname{Pr}\left\{T^{(p, 2)} \geq t_{\text {out }}^{p}\right\} \\
& =\operatorname{Pr}\left\{\tilde{B}_{p}\left[\ln \left(1+\frac{\mathrm{P}^{(p, 2)} \beta_{0}}{\mathrm{P}^{(s, 2)} h_{1}+N_{0}}\right)\right]^{-1} \geq t_{\text {out }}^{p}\right\} .
\end{aligned}
$$

Moreover, the condition of not degrading the PU performance is interpreted in terms of the timeout probability constraint as

$$
P_{\text {out }}^{(p, 2)} \leq \theta_{\text {th }}^{(p, 2)}
$$

where $\theta_{\mathrm{th}}^{(p, 2)}$ is the timeout probability constraint of the PU-Rx $x_{2}$.

\section{CCRN Communication}

In the first hop, the SU-Tx regulates its transmit power to send a packet of length $L_{s}$ bits to the SR. As the SR receives the packet successfully, it is stored in the buffer at the SR. Then, the SR feeds back a short ackowledgement (ACK) without delay to the SU-Tx. Upon the reception of the ACK, the SU-Tx transmits the next packet. The time consumed to send a packet in the first hop is given by

$$
T^{(s, 1)}=\frac{L_{s}}{B \log _{2}\left(1+\gamma^{(s, 1)}\right)} \triangleq \frac{\tilde{B}_{s}}{\ln \left(1+\gamma^{(s, 1)}\right)}
$$


where $\tilde{B}_{s}=L_{s} \ln (2) / B$, and $\gamma^{(s, 2)}$ is the SINR at the SR defined by

$$
\gamma^{(s, 1)}=\frac{\mathrm{P}^{(s, 1)} g_{0}}{\mathrm{P}^{(p, 1)} \alpha_{1}+N_{0}} .
$$

It is noted that the SU-Tx must control its transmit power $\mathrm{P}^{(s, 1)}$ to satisfy the timeout probability constraint $\theta_{\mathrm{th}}^{(p, 1)}$ of PU-Rx 1 given in (4). In addition, the SU-Tx transmit power is limited in practice; hence, it may be constrained by a peak transmit power $\mathrm{P}_{\mathrm{pk}}^{(s, 1)}$ as

$$
\mathrm{P}^{(s, 1)} \leq \mathrm{P}_{\mathrm{pk}}^{(s, 1)} .
$$

In other words, to send a packet to the SR, the SU-Tx should control its power to jointly meet the timeout probability constraint of the PU-Rx 1 given in (4) and the peak transmit power constraint of the SU-Tx given in (11).

In the second hop, the SR also adjusts its power to forward the packets in its buffer to the SU-Rx, where packets are served in first-come-first-serve order. It is noted that the buffer length of commercial devices is often very large nowadays. This fact can be adopted to assume that the length of the SR buffer is infinite. Similar to the first hop, the time it takes to transmit a packet in the second hop is expressed as

$$
T^{(s, 2)}=\frac{L_{s}}{B \log _{2}\left(1+\gamma^{(s, 2)}\right)} \triangleq \frac{\tilde{B}_{s}}{\ln \left(1+\gamma^{(s, 2)}\right)}
$$

where $\gamma^{(s, 2)}$ is the SINR at the SU-Rx and is given by

$$
\gamma^{(s, 2)}=\frac{\mathrm{P}^{(s, 2)} h_{0}}{\mathrm{P}^{(p, 2)} \beta_{1}+N_{0}} .
$$

The SR transmit power $\mathrm{P}^{(s, 2)}$ is also restricted by the timeout probability constraint of the $\mathrm{PU}-\mathrm{Rx}_{2}$ given in (8) and peak transmit power $\mathrm{P}_{\mathrm{pk}}^{(s, 2)}$ as

$$
\mathrm{P}^{(s, 2)} \leq \mathrm{P}_{\mathrm{pk}}^{(s, 2)} .
$$

Without loss of generality, each packet in the secondary or primary network is considered successfully transmitted if and only if the transmission time from the source to the intended receiver is less than a given timeout threshold. As a consequence, the event in which a packet is transmitted successfully is defined by

$$
T_{\text {suc }}^{(a, b)}=\left\{T^{(a, b)} \mid T^{(a, b)}<t_{\text {out }}^{a}\right\} .
$$

\section{E. Queueing Model for the SR Buffer}

We have assumed that all channels are constant for the duration of a packet transmission but may change independently thereafter. As a consequence, interarrival time of packets at the SR buffer is an identically and independently distributed (i.i.d.) RV with a general distribution, and so are the packet transmission times. Therefore, the traffic to the SR buffer can be modeled as a GI/G/1 queueing system [34], [35].

Let the interarrival time of packets and transmission time per packet be denoted by the RVs $A$ and $T$, respectively. Although an exact closed-form expression for the average waiting time of a packet in the buffer is not available, it can be approximated as [35]

$$
\mathbb{E}[W] \approx \frac{\rho \xi\left(C_{T}^{2}+C_{A}^{2}\right) \mathbb{E}[T]}{2(1-\rho)}
$$

where $W$ is an $\mathrm{RV}$ denoting the waiting time of a packet in the SR buffer, and

$$
\begin{aligned}
& \rho=\frac{\mathbb{E}[T]}{\mathbb{E}[A]}, \quad C_{X}^{2}=\frac{\operatorname{Var}[X]}{(\mathbb{E}[X])^{2}}, \quad X \in\{T, A\} \\
& \xi= \begin{cases}\exp \left(\frac{-2(1-\rho)\left(1-C_{A}^{2}\right)^{2}}{3 \rho\left(C_{T}^{2}+C_{A}^{2}\right)}\right), & C_{A}^{2}<1 \\
\exp \left(-(1-\rho) \frac{C_{A}^{2}-1}{C_{A}^{2}+4 C_{T}^{2}}\right), & C_{A}^{2} \geq 1 .\end{cases}
\end{aligned}
$$

According to [36], the queueing system is stable if and only if the average transmission time is less than the average interarrival time, i.e.,

$$
\rho=\frac{\mathbb{E}[T]}{\mathbb{E}[A]}<1
$$

where $\rho$ is the channel utilization.

\section{Performance Analysis}

Here, performance analysis for the considered system model is presented. We first consider adaptive transmit power policies for the SU-Tx and SR. Specifically, the cdf, pdf, and moments of the packet transmission time for the first and second hops are derived. Finally, performance in terms of end-to-end throughput, end-to-end transmission time, and stable condition for the SR operation are presented.

\section{A. Adaptive Transmit Power Policies}

Here, we derive the adaptive transmit power policies for the SU-Tx and the SR. Let us commence by considering a lemma as follows.

Lemma 1: Let $a, b, c$, and $d$ be positive constants. Further, let $X_{1}$ and $X_{2}$ be independent and exponentially distributed RVs with mean $\Omega_{1}$ and $\Omega_{2}$, respectively. Then, RV $Z$ defined as

$$
Z=d\left[\ln \left(1+\frac{a X_{1}}{b X_{2}+c}\right)\right]^{-1}
$$

has a cdf and a pdf, respectively, given by

$$
\begin{aligned}
F_{Z}(z)= & \frac{a \Omega_{1}}{b \Omega_{2}[\exp (d / z)-1]+a \Omega_{1}} \\
& \times \exp \left[-\frac{c}{a \Omega_{1}}\left(\exp \left(\frac{d}{z}\right)-1\right)\right] \\
f_{Z}(z)= & \left(\frac{a b d \Omega_{1} \Omega_{2}}{z^{2}\left[b \Omega_{2} \exp (d / z)+a \Omega_{1}-b \Omega_{2}\right]^{2}}\right. \\
& \left.\quad+\frac{c d}{z^{2}\left[b \Omega_{2} \exp (d / z)+a \Omega_{1}-b \Omega_{2}\right]}\right) \\
& \times \exp \left[\frac{d}{z}-\frac{c}{a \Omega_{1}} \exp \left(\frac{d}{z}\right)+\frac{c}{a \Omega_{1}}\right] .
\end{aligned}
$$


Proof: See Appendix A.

1) Adaptive Transmit Power Policy for the SU-Tx: The task of the SU-Tx is to select a transmit power level such that it can exploit the licensed spectrum of Region I as much as possible but does not cause harmful interference to the $\mathrm{PU}-\mathrm{Rx}_{1}$. Given the related constraints in (4) and (11), an adaptive transmit power policy for the SU-Tx is derived as follows.

From (3), the timeout probability of a packet transmitted by the PU-Tx 1 can be rewritten as

$$
P_{\text {out }}^{(p, 1)}=1-\operatorname{Pr}\left\{\tilde{B}_{p}\left[\ln \left(1+\frac{\mathrm{P}^{(p, 1)} \alpha_{0}}{\mathrm{P}^{(s, 1)} g_{1}+N_{0}}\right)\right]^{-1}<t_{\text {out }}^{p}\right\} .
$$

Applying Lemma 1 to (23), a closed-form expression for the $P_{\text {out }}^{(p, 1)}$ can be obtained as

$P_{\text {out }}^{(p, 1)}=1-\frac{\mathrm{P}^{(p, 1)} \Omega_{\alpha_{0}}}{\mathrm{P}^{(s, 1)} \Omega_{g_{1}} \Phi_{p}+\mathrm{P}^{(p, 1)} \Omega_{\alpha_{0}}} \exp \left(-\frac{N_{0} \Phi_{p}}{\mathrm{P}^{(p, 1)} \Omega_{\alpha_{0}}}\right)$

where $\Phi_{p}$ is defined as

$$
\Phi_{p}=\exp \left(\frac{\tilde{B}_{p}}{t_{\text {out }}^{p}}\right)-1 .
$$

Substituting (25) into (4), we obtain the maximum transmit power of the SU-Tx after some manipulations as

$$
\mathrm{P}^{(s, 1)}=\frac{\mathrm{P}^{(p, 1)} \Omega_{\alpha_{0}}}{\Omega_{g_{1}} \Phi_{p}} \chi_{1}
$$

where

$$
\chi_{1}=\max \left\{0, \frac{1}{1-\theta_{\mathrm{th}}^{(p, 1)}} \exp \left(-\frac{N_{0} \Phi_{p}}{\mathrm{P}^{(p, 1)} \Omega_{\alpha_{0}}}\right)-1\right\} .
$$

Combining (26) with (11), we can formulate an adaptive powerallocation policy for the SU-Tx as

$$
\mathcal{P}^{(s, 1)}=\min \left\{\frac{\mathrm{P}^{(p, 1)} \Omega_{\alpha_{0}}}{\Omega_{g_{1}} \Phi_{p}} \chi_{1}, \mathrm{P}_{\mathrm{pk}}^{(s, 1)}\right\} .
$$

2) Adaptive Transmit Power for the SR: Similar to the derivation for the first hop, the adaptive transmit power for the SR in the second hop is derived as follows.

From (8), the timeout probability of a packet transmitted by the PU-Tx ${ }_{2}$ is expressed as

$$
P_{\text {out }}^{(p, 2)}=1-\operatorname{Pr}\left\{\tilde{B}_{p}\left[\ln \left(1+\frac{\mathrm{P}^{(p, 2)} \beta_{0}}{\mathrm{P}^{(s, 2)} h_{1}+N_{0}}\right)\right]^{-1}<t_{\text {out }}^{p}\right\} .
$$

Using the same manipulations for the SU-Tx as earlier, an adaptive transmit power policy for the SR can be obtained as

$$
\mathcal{P}^{(s, 2)}=\min \left\{\frac{\mathrm{P}^{(p, 2)} \Omega_{\beta_{0}}}{\Omega_{h_{1}} \Phi_{p}} \chi_{2}, \mathrm{P}_{\mathrm{pk}}^{(s, 2)}\right\}
$$

where

$$
\chi_{2}=\max \left\{0, \frac{1}{1-\theta_{\mathrm{th}}^{(p, 2)}} \exp \left(-\frac{N_{0} \Phi_{p}}{\mathrm{P}^{(p, 2)} \Omega_{\beta_{0}}}\right)-1\right\} .
$$

\section{B. Performance Analysis for the First Hop}

1) Statistics for Packet Transmission Time in the First Hop: Here, we derive the timeout probability, the pdf of packet transmission time, and the first and second moments of packet transmission time from the SU-Tx to the SR.

In the first hop, the SU-Tx transmits packets to the SR by using the power-allocation policy given in (28). As such, the probability $P_{\text {out }}^{(s, 1)}$ that a packet is dropped due to timeout can be expressed as

$$
\begin{aligned}
P_{\text {out }}^{(s, 1)} & =\operatorname{Pr}\left\{T^{(s, 1)} \geq t_{\text {out }}^{s}\right\} \\
& =1-\operatorname{Pr}\left\{\tilde{B}_{s}\left[\ln \left(1+\frac{\mathcal{P}^{(s, 1)} g_{0}}{\mathrm{P}^{(p, 1)} \alpha_{1}+N_{0}}\right)\right]^{-1}<t_{\text {out }}^{s}\right\}
\end{aligned}
$$

where $t_{\text {out }}^{s}$ is the timeout threshold for the secondary network. Applying (21)-(32), we obtain a closed-form expression for the timeout probability $P_{\text {out }}^{(s, 1)}$ in the first hop as

$$
P_{\text {out }}^{(s, 1)}=\frac{\mathcal{P}^{(s, 1)} \Omega_{g_{0}}}{\mathrm{P}^{(p, 1)} \Omega_{\alpha_{1}} \Phi_{s}+\mathcal{P}^{(s, 1)} \Omega_{g_{0}}} \exp \left(-\frac{N_{0} \Phi_{s}}{\mathcal{P}^{(s, 1)} \Omega_{g_{0}}}\right)
$$

where $\Phi_{s}$ is defined as

$$
\Phi_{s}=\exp \left(\frac{\tilde{B}_{s}}{t_{\text {out }}^{s}}\right)-1 .
$$

In addition, the pdf of $T^{(s, 1)}$ can be easily derived by using (22) as in

$$
\begin{aligned}
& f_{T^{(s, 1)}}(x) \\
& =\left\{\frac{\mathcal{P}^{(s, 1)} \mathrm{P}^{(p, 1)} \tilde{B}_{s} \Omega_{g_{0}} \Omega_{\alpha_{1}}}{x^{2}\left[\mathrm{P}^{(p, 1)} \Omega_{\alpha_{1}} \exp \left(\tilde{B}_{s} / x\right)+\mathcal{P}^{(s, 1)} \Omega_{g_{0}}-\mathrm{P}^{(p, 1)} \Omega_{\alpha_{1}}\right]^{2}}\right. \\
& \left.\quad+\frac{N_{0} \tilde{B}_{s}}{x^{2}\left[\mathrm{P}^{(p, 1)} \Omega_{\alpha_{1}} \exp \left(\tilde{B}_{s} / x\right)+\mathcal{P}^{(s, 1)} \Omega_{g_{0}}-\mathrm{P}^{(p, 1)} \Omega_{\alpha_{1}}\right]}\right\} \\
& \quad \times \exp \left[\frac{\tilde{B}_{s}}{x}-\frac{N_{0}}{\mathcal{P}^{(s, 1)} \Omega_{g_{0}}} \exp \left(\frac{\tilde{B}_{s}}{x}\right)+\frac{N_{0}}{\mathcal{P}^{(s, 1)} \Omega_{g_{0}}}\right]
\end{aligned}
$$

and

$$
\begin{aligned}
& f_{T_{\text {suc }}^{(s, 1)}}(x) \\
& =\left\{\frac{\mathcal{P}^{(s, 1)} \mathrm{P}^{(p, 1)} \tilde{B}_{s} \Omega_{g_{0}} \Omega_{\alpha_{1}}}{x^{2}\left[\mathrm{P}^{(p, 1)} \Omega_{\alpha_{1}} \exp \left(\tilde{B}_{s} / x\right)+\mathcal{P}^{(s, 1)} \Omega_{g_{0}}-\mathrm{P}^{(p, 1)} \Omega_{\alpha_{1}}\right]^{2}}\right. \\
& \left.\quad+\frac{N_{0} \tilde{B}_{s}}{x^{2}\left[\mathrm{P}^{(p, 1)} \Omega_{\alpha_{1}} \exp \left(\tilde{B}_{s} / x\right)+\mathcal{P}^{(s, 1)} \Omega_{g_{0}}-\mathrm{P}^{(p, 1)} \Omega_{\alpha_{1}}\right]}\right\} \\
& \quad \times \frac{1}{1-\mathrm{P}_{\text {out }}^{(s, 1)}} \exp \left[\frac{\tilde{B}_{s}}{x}-\frac{N_{0}}{\mathcal{P}^{(s, 1)} \Omega_{g_{0}}} \exp \left(\frac{\tilde{B}_{s}}{x}\right)+\frac{N_{0}}{\mathcal{P}^{(s, 1)} \Omega_{g_{0}}}\right] .
\end{aligned}
$$


Furthermore, the probability for the event $T_{\text {suc }}^{(s, 1)}=$ $\left\{T^{(s, 1)} \mid T^{(s, 1)}<t_{\text {out }}^{s}\right\}$ that a packet from the SU-Tx to the SR is transmitted successfully can be expressed by using Bayes' rule as follows:

$$
\begin{aligned}
\operatorname{Pr}\left\{T^{(s, 1)} \mid T^{(s, 1)}<t_{\text {out }}^{s}\right\} & =\frac{\operatorname{Pr}\left\{T^{(s, 1)}, T^{(s, 1)}<t_{\text {out }}^{s}\right\}}{\operatorname{Pr}\left\{T^{(s, 1)}<t_{\text {out }}^{s}\right\}} \\
& =\frac{\operatorname{Pr}\left\{T^{(s, 1)}, T^{(s, 1)}<t_{\text {out }}^{s}\right\}}{1-P_{\text {out }}^{(s, 1)}}
\end{aligned}
$$

where $P_{\text {out }}^{(s, 1)}$ is given by (33). Hence, the cdf of $T_{\text {suc }}^{(s, 1)}$ can be formulated by using (37) as

$$
F_{T_{\mathrm{suc}}^{(s, 1)}}(x)=\frac{1}{1-P_{\mathrm{out}}^{(s, 1)}} \int_{0}^{x} f_{T^{(s, 1)}}(t) d t, \quad 0 \leq x<t_{\mathrm{out}}^{s} .
$$

Differentiating (38) with respect to $x$, we obtain the pdf of $T_{\text {suc }}^{(s, 1)}$ as

$$
f_{T_{\mathrm{suc}}^{(s, 1)}}(x)=\frac{f_{T^{(s, 1)}}(x)}{1-P_{\mathrm{out}}^{(s, 1)}}, \quad 0 \leq x<t_{\mathrm{out}}^{s}
$$

where $f_{T_{\mathrm{suc}}^{(s, 1)}}(x)$ is expressed in (36) and $f_{T_{\mathrm{suc}}^{(s, 1)}}(x)=0$, $x \geq t_{\text {out }}^{s}$.

Furthermore, we can determine the first and second moments of the successful packet transmission time as stated in Lemma 2.

Lemma 2: The first and second moments of $T_{\text {suc }}^{(s, 1)}$ are given by

$$
\mathbb{E}\left[\left(T_{\text {suc }}^{(s, 1)}\right)^{i}\right]=\frac{N_{0} \tilde{B}_{s} I_{1}(i)}{1-P_{\text {out }}^{(s, 1)}}+\frac{\mathcal{P}^{(s, 1)} \mathrm{P}^{(p, 1)} \tilde{B}_{s} \Omega_{g_{0}} \Omega_{\alpha_{1}} I_{2}(i)}{1-P_{\text {out }}^{(s, 1)}}
$$

where $i=1,2$, and $I_{k}(i), k=1,2$ is defined as

$$
I_{k}(i)=\int_{\Phi_{s}}^{\infty} \frac{\exp \left(-\frac{N_{0}}{\mathcal{P}^{(s, 1)} \Omega_{g_{0}}} t\right)}{\tilde{B}_{s}^{1-i}[\ln (t+1)]^{i}\left(\mathrm{P}^{(p, 1)} \Omega_{\alpha_{1}} t+\mathcal{P}^{(s, 1)} \Omega_{g_{0}}\right)^{k}} d t .
$$

Proof: See Appendix B.

Due to the impairments caused by the wireless channel, a packet may be transmitted successfully or unsuccessfully. As such, the moments of packet transmission time should be calculated based on the packet transmission time with and without being timed out [34]. Therefore, the moments of $T^{(s, 1)}$ can be obtained by using the law of total expectation as follows:

$\mathbb{E}\left[\left(T^{(s, 1)}\right)^{i}\right]=\left(1-P_{\text {out }}^{(s, 1)}\right) \mathbb{E}\left[\left(T_{\text {suc }}^{(s, 1)}\right)^{i}\right]+\left(t_{\text {out }}^{s}\right)^{i} P_{\text {out }}^{(s, 1)}$

where $\mathbb{E}\left[\left(T_{\text {suc }}^{(s, 1)}\right)^{i}\right]$ is given in Lemma 2.

2) Interarrival Time of Packets at the SR: As a packet is transmitted, the SU-Tx waits for an ACK. If the SU-Tx does not receive an ACK before $t_{\text {out }}^{s}$, the packet is considered lost or dropped. In this case, the packet is retransmitted, and the interarrival time of packets at the SR buffer can be expressed as

$$
A=T_{\text {suc }}^{(s, 1)}+\left(N^{(s, 1)}-1\right) t_{\text {out }}^{s}
$$

where $N^{(s, 1)}$ is a geometric RV that denotes the number of transmissions between two successful packet arrivals in the SR buffer. Its probability can be calculated as

$$
\operatorname{Pr}\left\{N^{(s, 1)}=\ell\right\}=\left(P_{\text {out }}^{(s, 1)}\right)^{\ell-1}\left(1-P_{\text {out }}^{(s, 1)}\right), \quad \ell \geq 1 .
$$

Additionally, we can derive the first and second moments of $N^{(s, 1)}$ as in the following Lemma 3.

Lemma 3: The first and second moments of the number of transmissions between two consecutive successful packet arrivals in the SR buffer can be calculated, respectively, as

$$
\begin{aligned}
\mathbb{E}\left[N^{(s, 1)}\right] & =\sum_{\ell=1}^{\infty} \ell \operatorname{Pr}\left\{N^{(s, 1)}=\ell\right\} \\
& =\sum_{\ell=1}^{\infty} \ell\left(P_{\text {out }}^{(s, 1)}\right)^{\ell-1}\left(1-P_{\text {out }}^{(s, 1)}\right) \\
& =\frac{1}{1-P_{\text {out }}^{(s, 1)}} \\
\mathbb{E}\left[\left(N^{(s, 1)}\right)^{2}\right] & =\sum_{\ell=1}^{\infty} \ell^{2} \operatorname{Pr}\left\{N^{(s, 1)}=\ell\right\} \\
& =\sum_{\ell=1}^{\infty} \ell^{2}\left(P_{\text {out }}^{(s, 1)}\right)^{\ell-1}\left(1-P_{\text {out }}^{(s, 1)}\right) \\
& =\frac{1+P_{\text {out }}^{(s, 1)}}{\left(1-P_{\text {out }}^{(s, 1)}\right)^{2}} .
\end{aligned}
$$

Proof: See Appendix C.

Lemma 4: The mean and variance of the interarrival time of packets at the SR buffer are, respectively, given by

$$
\begin{aligned}
\mathbb{E}[A]= & \frac{\mathcal{P}^{(s, 1)} \mathrm{P}^{(p, 1)} \tilde{B}_{s} \Omega_{g_{0}} \Omega_{\alpha_{1}} I_{2}(1)}{1-P_{\text {out }}^{(s, 1)}} \\
& +\frac{N_{0} \tilde{B}_{s} I_{1}(1)}{1-P_{\text {out }}^{(s, 1)}}+\frac{t_{\text {out }}^{s} P_{\text {out }}^{(s, 1)}}{1-P_{\text {out }}^{(s, 1)}} \\
\operatorname{Var}[A]= & \operatorname{Var}\left[T_{\text {suc }}^{(s, 1)}\right]+\frac{\left(t_{\text {out }}^{s}\right)^{2} P_{\text {out }}^{(s, 1)}}{\left(1-P_{\text {out }}^{(s, 1)}\right)^{2}}
\end{aligned}
$$

where $\mathbb{E}\left[\left(T_{\text {suc }}^{(s, 1)}\right)^{i}\right]$ is determined by (40).

Proof: See Appendix D.

\section{Performance Analysis for the Second Hop}

1) Statistics for Packet Transmission Time in the Second Hop: Similar to the first hop, in the second hop, the SR uses the adaptive transmit power policy given in (30) to transmit packets 
to the SU-Rx. The timeout probability for a packet transmitted in the second hop can be derived as

$$
\begin{aligned}
P_{\text {out }}^{(s, 2)} & =\operatorname{Pr}\left\{T^{(s, 2)} \geq t_{\text {out }}^{s}\right\} \\
& =1-\operatorname{Pr}\left\{\tilde{B}_{s}\left[\ln \left(1+\frac{\mathcal{P}^{(s, 2)} h_{0}}{\mathrm{P}^{(p, 2)} \beta_{1}+N_{0}}\right)\right]^{-1}<t_{\text {out }}^{s}\right\} \\
& =\frac{\mathcal{P}^{(s, 2)} \Omega_{h_{0}}}{\mathrm{P}^{(p, 2)} \Omega_{\beta_{1}} \Phi_{s}+\mathcal{P}^{(s, 2)} \Omega_{h_{0}}} \exp \left(-\frac{N_{0} \Phi_{s}}{\mathcal{P}^{(s, 2)} \Omega_{h_{0}}}\right) .
\end{aligned}
$$

We can now apply similar manipulations as in the first hop to derive the pdf and the moments of $T_{\text {suc }}^{(s, 2)}$ in the second hop as follows.

The pdf of packet transmission time without timeout $T_{\text {suc }}^{(s, 2)}$ can be given by (50) and $f_{T_{\text {suc }}^{(s, 2)}}(y)=0, y \geq t_{\text {out }}^{s}$, shown at the bottom of the page.

The first and second moments of $T^{(s, 2)}$ are obtained by

$\mathbb{E}\left[\left(T^{(s, 2)}\right)^{i}\right]=\left(1-P_{\text {out }}^{(s, 2)}\right) \mathbb{E}\left[\left(T_{\text {suc }}^{(s, 2)}\right)^{i}\right]+\left(t_{\text {out }}^{s}\right)^{i} P_{\text {out }}^{(s, 2)}$

where $i=1,2$, and $\mathbb{E}\left[\left(T_{\mathrm{suc}}^{(s, 2)}\right)^{i}\right]$ is formulated as

$\mathbb{E}\left[\left(T_{\text {suc }}^{(s, 2)}\right)^{i}\right]=\frac{N_{0} \tilde{B}_{s} J_{1}(i)+\mathcal{P}^{(s, 2)} \mathrm{P}^{(p, 2)} \tilde{B}_{s} \Omega_{h_{0}} \Omega_{\beta_{1}} J_{2}(i)}{1-P_{\text {out }}^{(s, 2)}}$

and $J_{k}(i), k=1,2$ is defined by

$$
J_{k}(i)=\int_{\Phi_{s}}^{\infty} \frac{\exp \left(-\frac{N_{0}}{\mathcal{P}^{(s, 2)} \Omega_{h_{0}}} t\right)}{\tilde{B}_{s}^{1-i}[\ln (t+1)]^{i}\left(\mathrm{P}^{(p, 2)} \Omega_{\beta_{1}} t+\mathcal{P}^{(s, 2)} \Omega_{h_{0}}\right)^{k}} d t .
$$

\section{Performance Measures}

Given the results in Section III-B and C, we are now in the position to derive performance measures for the CCRN such as the stable condition for the SR operation, end-toend throughput, and end-to-end average transmission time as follows.

Lemma 5: The SR operation is stable if and only if

$$
\begin{aligned}
& \frac{N_{0} \tilde{B}_{s} I_{1}(1)+\mathcal{P}^{(s, 1)} \mathrm{P}^{(p, 1)} \tilde{B}_{s} \Omega_{g_{0}} \Omega_{\alpha_{1}} I_{2}(1)+t_{\text {out }}^{s} P_{\text {out }}^{(s, 1)}}{1-P_{\text {out }}^{(s, 1)}} \\
& >N_{0} \tilde{B}_{s} J_{1}(1)+\mathcal{P}^{(s, 2)} \mathrm{P}^{(p, 2)} \tilde{B}_{s} \Omega_{h_{0}} \Omega_{\beta_{1}} J_{2}(1)+t_{\text {out }}^{s} P_{\text {out }}^{(s, 2)} .
\end{aligned}
$$

Proof: Because the interarrival time of packets is i.i.d. with a general distribution, the transmission time of packets is also i.i.d. The packet traffic at the SR can be modeled as a GI/G/1 queueing system. Using the stable condition given in (19), we can deduce the desired stable condition for the SR buffer as

$$
\mathbb{E}[A]>\mathbb{E}\left[T^{(s, 2)}\right]
$$

Substituting (47) and (51) for $i=1$ into (55), we obtain (54).

The end-to-end throughput for the considered CCRN can be determined by the following Lemma 6.

Lemma 6: The throughput of the considered CCRN in the stable and unstable transmission condition can be formulated as

$$
R=\frac{1-P_{\mathrm{out}}^{(s, 2)}}{\max \left\{\mathbb{E}\left[T^{(s, 2)}\right], \mathbb{E}[A]\right\}} \text { (packets/s). }
$$

Proof: If the SR operation is stable, i.e., $\mathbb{E}[A]>$ $\mathbb{E}\left[T^{(s, 2)}\right]$, then the average transmission rate of the $\mathrm{SR}$ is equal to the average arrival rate of packets at the SR buffer. Hence, the end-to-end throughput can be determined as

$$
\frac{1}{\mathbb{E}[A]}=\frac{1}{\max \left\{\mathbb{E}\left[T^{(s, 2)}\right], \mathbb{E}[A]\right\}}
$$

On the other hand, if the SR operation is unstable, i.e., $\mathbb{E}[A] \leq \mathbb{E}\left[T^{(s, 2)}\right]$, then the average transmission rate of the SR is equal to the average transmission rate of the $\mathrm{SR} \rightarrow \mathrm{SU}-\mathrm{Rx}$ link because the SR becomes a bottleneck. Hence, the average transmission rate of the SR can be expressed as

$$
\frac{1}{\mathbb{E}\left[T^{(s, 2)}\right]}=\frac{1}{\max \left\{\mathbb{E}\left[T^{(s, 2)}\right], \mathbb{E}[A]\right\}}
$$

Moreover, in both cases (stable and unstable condition), a fraction of $P_{\text {out }}^{(s, 2)}$ of packets is dropped due to timeout in the $\mathrm{SR} \rightarrow \mathrm{SU}-\mathrm{Rx}$ link. Therefore, the SU-Rx receives packets at an average rate, as given in (56).

Finally, the end-to-end transmission time from the SU-Tx to the SU-Rx over the SR buffer can be calculated as

$$
D=\mathbb{E}\left[T_{\text {suc }}^{(s, 1)}\right]+\mathbb{E}[W]+\mathbb{E}\left[T_{\text {suc }}^{(s, 2)}\right]
$$

where $\mathbb{E}\left[T_{\text {suc }}^{(s, 1)}\right]$ and $\mathbb{E}\left[T_{\text {suc }}^{(s, 2)}\right]$ are given, respectively, in (40) and (52) for $i=1$. The average waiting time of a packet in the

$$
\begin{array}{r}
f_{T_{\mathrm{suc}}^{(s, 2)}}(y)=\left\{\frac{\mathcal{P}^{(s, 2)} \mathrm{P}^{(p, 2)} \tilde{B}_{s} \Omega_{h_{0}} \Omega_{\beta_{1}}}{y^{2}\left[\mathrm{P}^{(p, 2)} \Omega_{\beta_{1}} \exp \left(\tilde{B}_{s} / y\right)+\mathcal{P}^{(s, 2)} \Omega_{h_{0}}-\mathrm{P}^{(p, 2)} \Omega_{\beta_{1}}\right]^{2}}+\frac{N_{0} \tilde{B}_{s}}{y^{2}\left[\mathrm{P}^{(p, 2)} \Omega_{\beta_{1}} \exp \left(\tilde{B}_{s} / y\right)+\mathcal{P}^{(s, 2)} \Omega_{h_{0}}-\mathrm{P}^{(p, 2)} \Omega_{\beta_{1}}\right]}\right\} \\
\times \frac{1}{1-P_{\text {out }}^{(s, 2)}} \exp \left[\frac{\tilde{B}_{s}}{y}-\frac{N_{0}}{\mathcal{P}^{(s, 2)} \Omega_{h_{0}}} \exp \left(\frac{\tilde{B}_{s}}{y}\right)+\frac{N_{0}}{\mathcal{P}^{(s, 2)} \Omega_{h_{0}}}\right], \quad 0 \leq y<t_{\text {out }}^{s}
\end{array}
$$




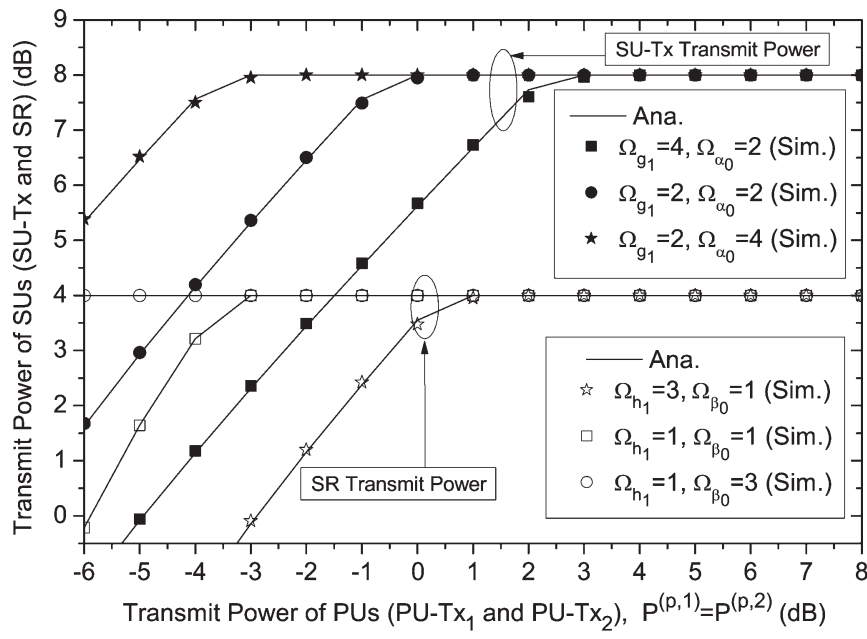

Fig. 2. Impact of the channel mean power on the adaptive transmit power of the SUs $\left(\mathcal{P}^{(s, 1)}\right.$ and $\mathcal{P}^{(s, 2)}$ ), in which the peak transmit power of the SUTx and SR are set, respectively, to $\mathrm{P}_{\mathrm{pk}}^{(s, 1)}=8 \mathrm{~dB}$ and $\mathrm{P}_{\mathrm{pk}}^{(s, 2)}=4 \mathrm{~dB}$, and $\Omega_{g_{0}}=\Omega_{h_{0}}=\Omega_{\beta_{1}}=\Omega_{\alpha_{1}}=2$.

SR buffer $\mathbb{E}[W]$ is given by

$$
\mathbb{E}[W] \approx \frac{\rho \xi\left(C_{T^{(s, 2)}}^{2}+C_{A}^{2}\right) \mathbb{E}\left[T^{(s, 2)}\right]}{2(1-\rho)}
$$

where

$$
\begin{array}{ll}
\rho=\frac{\mathbb{E}\left[T^{(s, 2)}\right]}{\mathbb{E}[A]}, \quad C_{X}^{2}=\frac{\mathbf{V} \operatorname{ar}[X]}{(\mathbb{E}[X])^{2}}, \quad X \in\left\{T^{(s, 2)}, A\right\} \\
\xi= \begin{cases}\exp \left(\frac{-2(1-\rho)\left(1-C_{A}^{2}\right)^{2}}{3 \rho\left(C_{T^{(s, 2)}}^{2}+C_{A}^{2}\right)}\right), & C_{A}^{2}<1 \\
\exp \left(-(1-\rho) \frac{C_{A}^{2}-1}{C_{A}^{2}+4 C_{T}^{2}(s, 2)}\right), & C_{A}^{2} \geq 1 .\end{cases}
\end{array}
$$

\section{NumERICAL RESUltS}

Here, we present simulation and analysis results for the considered system. In particular, we study the impact of the PU-Tx transmit power and the channel mean power on the end-to-end throughput, end-to-end average packet transmission time, and stable condition for the SR operation. Unless otherwise stated, the following system parameters are used for both simulation and analysis.

System bandwidth: $B=2 \mathrm{MHz}$.

Packet size: $L_{s}=L_{p}=224$ bits (28 bytes).

Timeout: $t_{\text {out }}^{s}=t_{\text {out }}^{p}=0.06 \mathrm{~s}$.

Outage constraints: $\theta_{\text {out }}^{(p, 1)}=\theta_{\text {out }}^{(p, 2)}=1 \%$.

\section{A. Adaptive Transmit Power}

Fig. 2 shows the SUs transmit power $\left(\mathcal{P}^{(s, 1)}\right.$ and $\left.\mathcal{P}^{(s, 2)}\right)$ as a function of the PU-Tx transmit power $\left(\mathrm{P}^{(p, 1)}\right.$ and $\left.\mathrm{P}^{(p, 2)}\right)$ for different channel mean power values. As expected, when the channel mean power value of the interference links from the secondary network to the primary network increases (e.g., $\Omega_{g_{1}}=2$ to 4 or $\Omega_{h_{1}}=1$ to 3 ) and the PUs operate in the

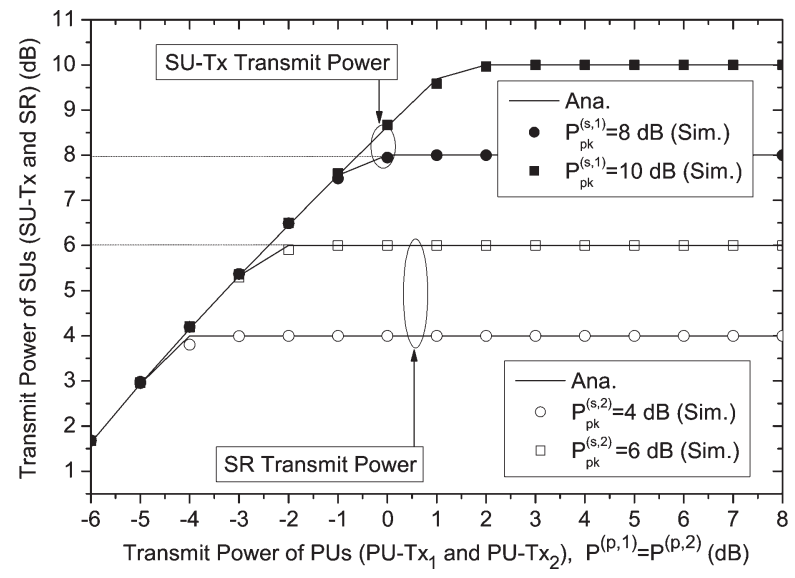

Fig. 3. Impact of the transmit power of the $\mathrm{PU}^{-\mathrm{Tx}_{1}}\left(\mathrm{P}^{(p, 1)}\right)$ and $\mathrm{PU}_{-\mathrm{Tx}_{2}}\left(\mathrm{P}^{(p, 2)}\right)$ on the adaptive transmit power of the SU-Tx $\left(\mathcal{P}^{(s, 1)}\right)$ and SR $\left(\mathcal{P}^{(s, 2)}\right)$, respectively, with identical channel mean power, i.e., $\Omega_{\alpha_{0}}=$ $\Omega_{\alpha_{1}}=\Omega_{h_{0}}=\Omega_{h_{1}}=\Omega_{\beta_{0}}=\Omega_{\beta_{1}}=\Omega_{g_{0}}=\Omega_{g_{1}}=2$.

low transmit power regime, the transmit power of the SUs decreases significantly. In other words, when the channel mean power of these interference links is high, the PU-Rxs become subject to stronger interference from the SUs. Accordingly, the SUs must decrease their transmit power to satisfy the outage constraint of the PUs. Interestingly, we can see that, as the channel mean power of the PU communication links increases (e.g., $\Omega_{\alpha_{0}}=2$ to 4 or $\Omega_{\beta_{0}}=1$ to 3 ), the SUs transmit power can be increased significantly. This can be explained by the fact that the packet timeout probability of the PUs decreases as the channel mean power of the PUs increases. Thus, the PUs can tolerate more interference from the SUs, i.e., the SUs can increase their transmit power and still satisfy the outage constraint of the PUs. This result also means that the channel mean power of the PU communication links should be utilized such that the performance of the secondary network can be enhanced.

Fig. 3 plots the SUs (SU-Tx and SR) transmit power as a function of the PU-Txs (PU-Tx $x_{1}$ and PU-Tx ${ }_{2}$ ) transmit power, respectively, for different $\mathrm{SU}$ peak transmit power values. We can see that, as the transmit power of the PU-Txs increases, the transmit power of SUs also increases. However, as the transmit power of PU-Txs increases further, the transmit power of SUs is saturated at specific values, e.g., the SU-Tx transmit power is saturated at $\mathcal{P}^{(s, 1)}=8 \mathrm{~dB}$ given $\mathrm{P}_{\mathrm{pk}}^{(s, 1)}=8 \mathrm{~dB}$, whereas the SR transmit power is saturated at $\mathcal{P}^{(s, 2)}=6 \mathrm{~dB}$ given $\mathrm{P}_{\mathrm{pk}}^{(s, 2)}=6 \mathrm{~dB}$. These results are in line with the $\mathrm{SU}$ transmit power policies $\left(\mathcal{P}^{(s, 1)}\right.$ and $\left.\mathcal{P}^{(s, 2)}\right)$ given in (28) and (30), respectively, where the transmit power of the SU-Tx and SR is bounded.

\section{B. End-to-End Throughput of the CCRN}

Fig. 4 shows the throughput as a function of $\mathrm{PU}_{-} \mathrm{Tx}_{2}$ transmit power for different values of the SR peak transmit power $\mathrm{P}_{\mathrm{pk}}^{(s, 2)}=1,3,4,8 \mathrm{~dB}$ and identical channel mean power $\Omega_{\alpha_{0}}=$ $\Omega_{\alpha_{1}}=\Omega_{h_{0}}=\Omega_{h_{1}}=\Omega_{\beta_{0}}=\Omega_{\beta_{1}}=\Omega_{g_{0}}=\Omega_{g_{1}}=2$. For brevity, we discuss only the scenario where the $\mathrm{PU}_{-} \mathrm{Tx}_{1}$ transmit power 


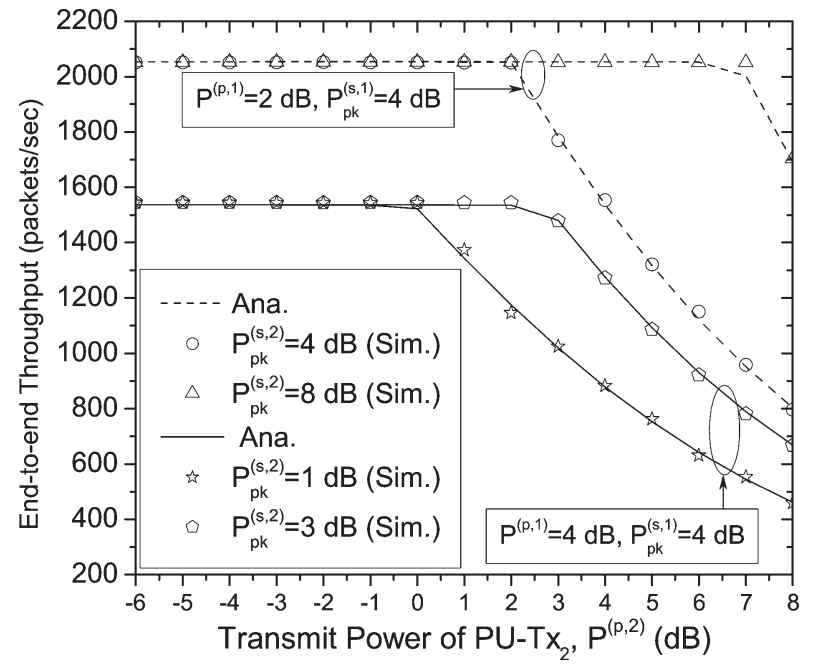

Fig. 4. Impact of the PU-Tx 2 transmit power in Region II, i.e., $\mathrm{P}^{(p, 2)}$, on the throughput of the CCRN for identical channel mean power, i.e., $\Omega_{\alpha_{0}}=$ $\Omega_{\alpha_{1}}=\Omega_{h_{0}}=\Omega_{h_{1}}=\Omega_{\beta_{0}}=\Omega_{\beta_{1}}=\Omega_{g_{0}}=\Omega_{g_{1}}=2$.

$\mathrm{P}^{(p, 1)}=2 \mathrm{~dB}$ and the SU-Tx peak transmit power $\mathrm{P}_{\mathrm{pk}}^{(s, 1)}=4 \mathrm{~dB}$ in the first hop and distinguish the following cases.

- Case 1: SR peak transmit power is set to $\mathrm{P}_{\mathrm{pk}}^{(s, 2)}=4 \mathrm{~dB}$.

- Case 2: SR peak transmit power is set to $\mathrm{P}_{\mathrm{pk}}^{(s, 2)}=8 \mathrm{~dB}$.

The other cases, with $\mathrm{P}^{(p, 1)}=4 \mathrm{~dB}$ and $\mathrm{P}_{\mathrm{pk}}^{(s, 1)}=4 \mathrm{~dB}$ where the SR peak transmit power is given as 1 and $3 \mathrm{~dB}$, show a similar progression of the end-to-end throughput versus transmit power of the PU- $\mathrm{Tx}_{2}$.

As shown in the figure, the simulation and analytical results match very well. In all cases, the throughput is first kept at a constant level in the low regime of the $\mathrm{PU}_{-\mathrm{Tx}_{2}}$ transmit power $\mathrm{P}^{(p, 2)}$ and then decreases in the high regime of $\mathrm{P}^{(p, 2)}$. In particular, for Case 1, the throughput is unchanged for $\mathrm{P}^{(p, 2)}<2 \mathrm{~dB}$, and it is degraded for $\mathrm{P}^{(p, 2)}>2 \mathrm{~dB}$. These results are thought to be due to the fact that, in the low regime of the PU-Tx 2 transmit power, the PU-Tx 2 does not cause much interference to the SU-Rx. Thus, the SR can adapt its transmit power $\mathcal{P}^{(s, 2)}$ to the change of $\mathrm{P}^{(p, 2)}$, following the strategy given in (30). However, in the high regime of the $\mathrm{PU}_{-\mathrm{TX}_{2}}$ transmit power, the SR cannot increase its transmit power further due to the peak transmit power constraint. As a consequence, the $\mathrm{PU}_{-} \mathrm{Tx}_{2}$ transmit power now becomes strong interference to the SU-Rx. This results in a large increase in the number of dropped packets due to timeout in the second hop, which degrades the end-to-end throughput of the CCRN. On the other hand, in Case 2, the throughput becomes more stable in the high regime of the $\mathrm{PU}-\mathrm{Tx}_{2}$ transmit power and is only degraded as the PU-Tx 2 increases beyond $6 \mathrm{~dB}$. In other words, if the SR peak transmit power $\mathrm{P}_{\mathrm{pk}}^{(s, 2)}$ is set to a high value, the $\mathrm{SR} \rightarrow \mathrm{SU}-\mathrm{Rx}$ link in the second hop may not become a bottleneck as the PU-Tx 2 transmit power increases to a high value.

Fig. 5 shows the impact of channel mean power of the interference links on the CCRN throughput. In particular, we have set identical channel mean power for all communication links of both the primary network and the secondary network,

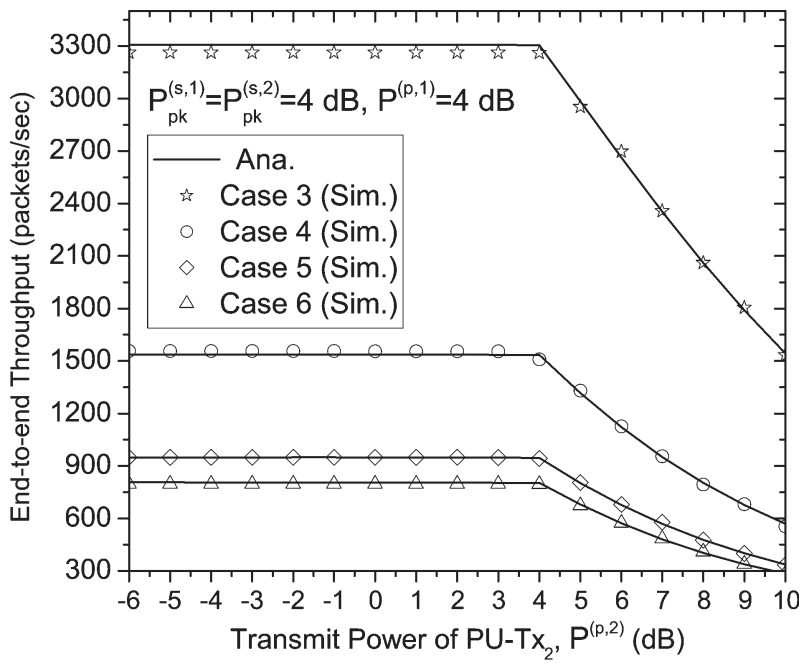

Fig. 5. Impact of the $\mathrm{PU}_{-} \mathrm{Tx}_{2}$ transmit power $\mathrm{P}^{(p, 2)}$ on the throughput of the CCRN for different channel mean power values of interference links. Case 3: $\Omega_{h_{1}}=\Omega_{g_{1}}=\Omega_{\beta_{1}}=\Omega_{\alpha_{1}}=0.5$. Case 4: $\Omega_{h_{1}}=\Omega_{g_{1}}=\Omega_{\beta_{1}}=$ $\Omega_{\alpha_{1}}=2$. Case 5: $\Omega_{h_{1}}=\Omega_{g_{1}}=\Omega_{\beta_{1}}=\Omega_{\alpha_{1}}=4$. Case 6: $\Omega_{h_{1}}=\Omega_{g_{1}}=$ 0.5 and $\Omega_{\beta_{1}}=\Omega_{\alpha_{1}}=5$.

i.e., $\Omega_{g_{0}}=\Omega_{h_{0}}=\Omega_{\beta_{0}}=\Omega_{\alpha_{0}}=2$. Then, the following four cases are considered:

- Case 3: The primary network and the secondary network do not cause severe interference to each other due to low channel mean power of the interference links, i.e., $\Omega_{h_{1}}=$ $\Omega_{g_{1}}=\Omega_{\beta_{1}}=\Omega_{\alpha_{1}}=0.5$.

- Case 4: This case shall be considered a reference where all channel mean power values for interference links and communication links are identical, i.e., $\Omega_{h_{1}}=\Omega_{g_{1}}=\Omega_{\beta_{1}}=$ $\Omega_{\alpha_{1}}=2$.

- Case 5: The primary network and the secondary network can cause strong interference to each other due to high channel mean power of the interference links, i.e., $\Omega_{h_{1}}=$ $\Omega_{g_{1}}=\Omega_{\beta_{1}}=\Omega_{\alpha_{1}}=4$.

- Case 6: The primary network causes strong interference to the secondary network due to high channel mean power of the PU-Tx $1 \rightarrow$ SR and PU-Tx $2 \rightarrow$ SU-Rx interference links, i.e., $\Omega_{\beta_{1}}=\Omega_{\alpha_{1}}=5$, whereas the secondary network causes weak interference to the primary network due to low channel mean power of the SU $\mathrm{Tx} \rightarrow \mathrm{PU}^{-\mathrm{Rx}_{1}}$ and $\mathrm{SR} \rightarrow \mathrm{PU}^{-\mathrm{Rx}_{2}}$ interference links, i.e., $\Omega_{h_{1}}=\Omega_{g_{1}}=0.5$.

We can observe in Fig. 5 that the throughput of Case 3 outperforms the reference Case 4 . This is because the interference between the primary network and the secondary network is reduced as the channel mean power of the interference links is decreased from $\Omega_{h_{1}}=\Omega_{g_{1}}=\Omega_{\beta_{1}}=\Omega_{\alpha_{1}}=2$ to $\Omega_{h_{1}}=$ $\Omega_{g_{1}}=\Omega_{\beta_{1}}=\Omega_{\alpha_{1}}=0.5$. Accordingly, the timeout probability constraint of the primary network may not be violated. Thus, the SU-Tx and the SR can increase their transmit power to reduce the packet transmission time. As such, the number of packets that suffer a timeout in the secondary network may be decreased; hence, the end-to-end throughput of the CCRN is increased. This special case of having poor interference links can be related, for example, to the scenario where operating frequencies are switched between the two hops of the secondary 


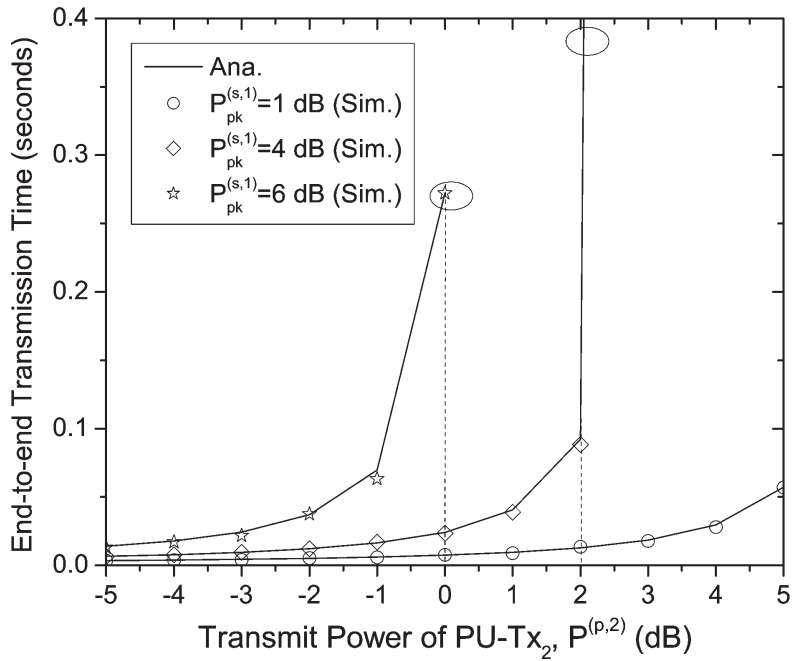

Fig. 6. Impact of the PU-Tx $x_{2}$ transmit power in Region II, i.e., $\mathrm{P}^{(p, 2)}$, on the end-to-end transmission time of packets for identical channel mean power with $\Omega_{\alpha_{0}}=\Omega_{\alpha_{1}}=\Omega_{h_{0}}=\Omega_{h_{1}}=\Omega_{\beta_{0}}=\Omega_{\beta_{1}}=\Omega_{g_{0}}=\Omega_{g_{1}}=2$, i.e., $\mathrm{P}^{(p, 1)}=\mathrm{P}_{\mathrm{pk}}^{(s, 2)}=4 \mathrm{~dB}$.

network to reduce the mutual interference between the primary and secondary networks, as well as to increase the system performance.

In Case 5, where the channel mean power of the interference links is set to high values, i.e., $\Omega_{h_{1}}=\Omega_{g_{1}}=\Omega_{\beta_{1}}=\Omega_{\alpha_{1}}=4$, the interference between the primary network and the secondary network increases as well. Accordingly, the timeout probability constraints of the primary network may be easily violated with low power levels of the SU-Tx and SR. Therefore, the SU-Tx and SR must reduce their transmit power to protect the communication of the primary network. As a consequence, the packet transmission time and the number of packets being timed out increase. Therefore, the throughput of Case 5 is lower than that of Case 4.

Finally, Case 6 results in the worst performance compared with the other considered cases. This can be explained by the fact that the SR and SU-Rx suffer from strong interference caused by the PU-Tx $x_{1}$ and PU-Tx 2 , respectively. Therefore, there may be many packets in both hops of the CCRN being timed out, which leads to low end-to-end throughput.

\section{End-to-End Transmission Time}

In Fig. 6, the end-to-end transmission time is plotted as a function of the $\mathrm{PU}_{-} \mathrm{Tx}_{2}$ transmit power $\mathrm{P}^{(p, 2)}$ for different values of the SU-Tx peak transmit power in the first hop, i.e., $\mathrm{P}_{\mathrm{pk}}^{(s, 1)}=1,4,6 \mathrm{~dB}$. It is shown in the figure that the end-toend transmission time increases as the PU- $\mathrm{Tx}_{2}$ transmit power increases. Typically, the end-to-end transmission time increases rapidly as the SU-Tx peak transmit power is set to a high value such as $\mathrm{P}_{\mathrm{pk}}^{(s, 1)}=4,6 \mathrm{~dB}$. This can be understood by the fact that, as the SU-Tx peak transmit power is set to a high value, e.g., $\mathrm{P}_{\mathrm{pk}}^{(s, 1)}=6 \mathrm{~dB}$, the SU-Tx can adjust its transmit power following the policy given in (28) to send more packets to the SR, i.e., the number of packets arriving at the SR buffer increases. On the other hand, in the second hop, increasing

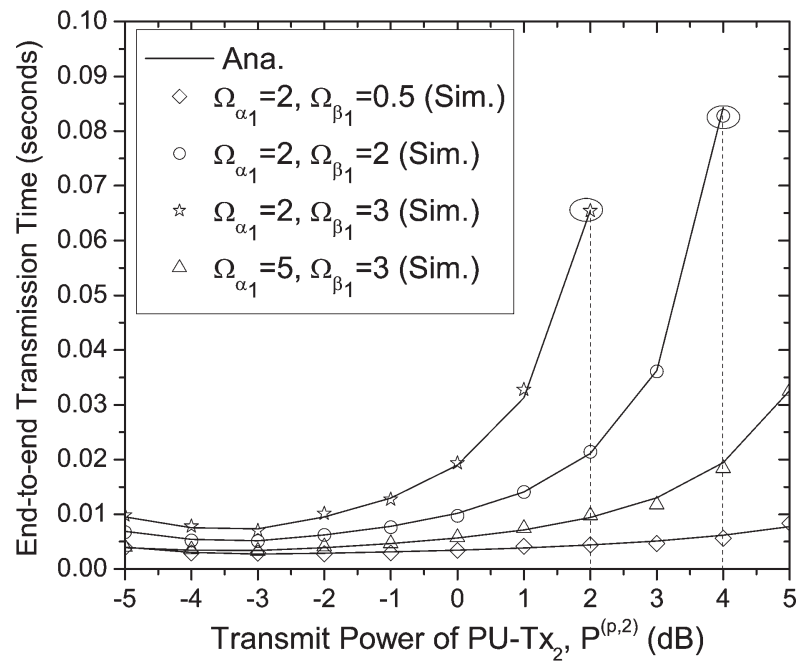

Fig. 7. Impact of the PU-Tx $x_{2}$ transmit power $\mathrm{P}^{(p, 2)}$ on the end-to-end transmission time of packets for channel mean power with $\Omega_{\alpha_{0}}=\Omega_{g_{0}}=$ $\Omega_{g_{1}}=\Omega_{h_{0}}=\Omega_{\beta_{0}}=\Omega_{h_{1}}=2$, and $\mathrm{P}^{(p, 1)}=\mathrm{P}_{\mathrm{pk}}^{(s, 1)}=\mathrm{P}_{\mathrm{pk}}^{(s, 2)}=6 \mathrm{~dB}$.

PU-Tx 2 transmit power $\mathrm{P}^{(p, 2)}$ leads to a decrease in the SURx SINR given in (13). Hence, the packet transmission time is increased. As a consequence, the number of packets arriving at the SR buffer increases, whereas the number of packets leaving the SR buffer decreases. Therefore, the number of packets waiting in the SR buffer and the end-to-end transmission time increase.

Fig. 7 shows the end-to-end transmission time as a function of the PU- $\mathrm{Tx}_{2}$ transmit power $\mathrm{P}^{(p, 2)}$ for different channel mean power values of the $\mathrm{PU}_{-\mathrm{Tx}_{1}} \rightarrow \mathrm{SR}$ and $\mathrm{PU}_{-\mathrm{Tx}_{2}} \rightarrow \mathrm{SU}-\mathrm{Rx}$ interference links. In particular, we set the channel mean power $\Omega_{g_{0}}=\Omega_{h_{0}}=\Omega_{\beta_{0}}=\Omega_{\alpha_{0}}=\Omega_{h_{1}}=\Omega_{g_{1}}=2$ and consider the following two cases:

- Case 7: The channel mean power of the PU-Tx $\mathrm{T}_{1} \rightarrow \mathrm{SR}$ interference link is set to $\Omega_{\alpha_{1}}=2$, whereas the channel mean power of the PU-Tx $x_{2} \rightarrow$ SU-Rx interference link is set to $\Omega_{\beta_{1}}=0.5,2,3$.

- Case 8: The channel mean power of the PU-Tx $x_{2} \rightarrow \mathrm{SU}-\mathrm{Rx}$ interference link is set to $\Omega_{\beta_{1}}=3$, whereas the channel mean power of the $\mathrm{PU}-\mathrm{Tx}_{1} \rightarrow \mathrm{SR}$ interference link is set to $\Omega_{\alpha_{1}}=2,5$.

As expected, the end-to-end transmission time increases when increasing the PU-Tx $x_{2}$ transmit power $\mathrm{P}^{(p, 2)}$. Moreover, for Case 7, we can observe that the end-to-end transmission time increases as the channel mean power of the PU-Tx $x_{2} \rightarrow$ SU-Rx interference link increases from $\Omega_{\beta_{1}}=0.5$ to $\Omega_{\beta_{1}}=3$. For the same reason as for the throughput results, the SU-Rx may be subject to strong interference from the PU-Tx 2 when the channel mean power of the PU-Tx $x_{2} \rightarrow$ SU-Rx interference link is high, e.g., $\Omega_{\beta_{1}}=3$. Accordingly, the SU-Rx SINR given in (13) decreases and packet transmission time in the second hop increases. As such, the remaining packets have to stay in the SR buffer for a long time, leading to an increase in end-to-end transmission time.

In Case 8, the end-to-end transmission time decreases as the channel mean power of the PU-Tx $x_{1} \rightarrow$ SR interference link in the first hop increases from $\Omega_{\alpha_{1}}=2$ to $\Omega_{\alpha_{1}}=5$. In this case, 


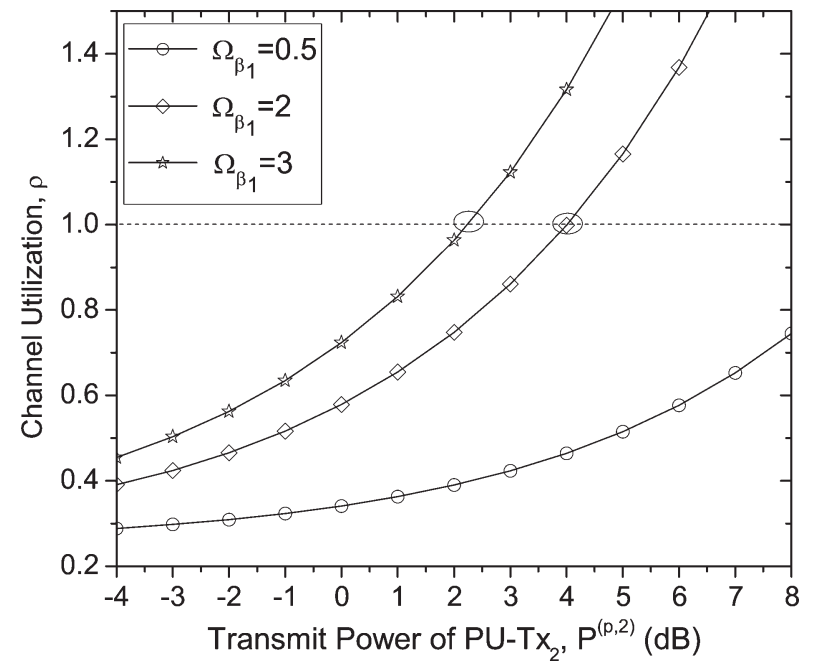

Fig. 8. Impact of channel mean power on the stability of the SR operation with $\Omega_{g_{0}}=\Omega_{g_{1}}=\Omega_{h_{0}}=\Omega_{h_{1}}=\Omega_{\beta_{0}}=\Omega_{\alpha_{0}}=\Omega_{\alpha_{1}}=2$ and $\mathrm{P}^{(p, 1)}=$ $\mathrm{P}_{\mathrm{pk}}^{(s, 1)}=\mathrm{P}_{\mathrm{pk}}^{(s, 2)}=4 \mathrm{~dB}$.

the SR is subject to strong interference from the PU-Tx ${ }_{1}$, and the SR SINR given in (10) decreases. Therefore, the packet transmission time in the first hop increases. As a consequence, the number of packets arriving at the SR buffer decreases, which in turn reduces the waiting time in the SR buffer and the end-to-end transmission time.

\section{Stability of the SR Operation}

Finally, we examine the impact of the channel mean power of the PU-Tx $x_{2} \rightarrow$ SU-Rx interference link on the stability of the SR operation, as shown in Fig. 8. As expected, the SR operation with the low channel mean power of the PU-Tx $2 \rightarrow$ SU-Rx interference link $\Omega_{\beta_{1}}=0.5$ is more stable than for the higher channel mean power. Specifically, the channel utilization $\rho$ is kept below 1 for the entire range of the considered PU-Tx 2 transmit power. On the other hand, the SR operation becomes unstable $(\rho \geq 1)$ for $\Omega_{\beta_{1}}=2,3$ once the PU-Tx 2 transmit power progresses toward high values, e.g., $\mathrm{P}^{(p, 2)}>4 \mathrm{~dB}$. In the case of these high channel mean power values, the PU-Tx ${ }_{2}$ causes strong interference to the SU-Rx when the PU-Tx ${ }_{2}$ transmit power $\mathrm{P}^{(p, 2)}$ increases. Hence, the packet transmission time increases in the second hop, which leads the channel utilization $\rho=\mathbb{E}\left[T^{(s, 2)}\right] / \mathbb{E}[A]$ to become greater than 1 , causing the SR operation to be unstable, as in (63)-(66), shown at the bottom of the page.

\section{CONCLUSION}

In this paper, we have analyzed the performance of a CCRN with a buffered relay. In particular, we have assumed that packets in the primary network and secondary network are subject to timeout constraints. In addition, we have assumed that the communication channels and interference channels undergo Rayleigh fading. The transmit power of the SU-Tx and SR is subject to both the peak transmit power constraint and the timeout probability constraint of the PUs. On this basis, adaptive transmit power policies for the SU-Tx and the SR have been investigated. The cdf, pdf, timeout probability, and moments for packet transmission time in each hop have been derived. Moreover, by employing the GI/G/1 queueing model, the performance analysis in terms of the end-to-end throughput, end-to-end transmission time, and stable condition for the SR operation has been provided for the considered system. Numerical results have been presented to quantify the impact of PU-Tx transmit power and channel mean power of the interference links on the performance of the CCRN.

$$
\begin{aligned}
& F_{Z}(z)=\frac{a \Omega_{1}}{b \Omega_{2}[\exp (d / z)-1]+a \Omega_{1}} \exp \left[-\frac{c}{a \Omega_{1}}\left(\exp \left(\frac{d}{z}\right)-1\right)\right] \\
& f_{Z}(z)=\left(\frac{a b d \Omega_{1} \Omega_{2}}{z^{2}\left[b \Omega_{2} \exp (d / z)+a \Omega_{1}-b \Omega_{2}\right]^{2}}+\frac{c d}{z^{2}\left[b \Omega_{2} \exp (d / z)+a \Omega_{1}-b \Omega_{2}\right]}\right) \\
& \times \exp \left[\frac{d}{z}-\frac{c}{a \Omega_{1}} \exp (d / z)+\frac{c}{a \Omega_{1}}\right] \\
& \mathbb{E}\left[\left(T_{\text {suc }}^{(s, 1)}\right)^{i}\right]=\frac{\mathcal{P}^{(s, 1)} \mathrm{P}^{(p, 1)} \tilde{B}_{s} \Omega_{g_{0}} \Omega_{\alpha_{1}}}{1-P_{\text {out }}^{(s, 1)}} \int_{0}^{t_{\text {out }}^{s}} \frac{\exp \left[\frac{\tilde{B}_{s}}{x}-\frac{N_{0}}{\mathcal{P}^{(s, 1)} \Omega_{g_{0}}} \exp \left(\frac{\tilde{B}_{s}}{x}\right)+\frac{N_{0}}{\mathcal{P}^{(s, 1)} \Omega_{g_{0}}}\right]}{x^{2-i}\left[\mathrm{P}^{(p, 1)} \Omega_{\alpha_{1}} \exp \left(\frac{\tilde{B}_{s}}{x}\right)+\mathcal{P}^{(s, 1)} \Omega_{g_{0}}-\mathrm{P}^{(p, 1)} \Omega_{\alpha_{1}}\right]^{2}} d x \\
& +\frac{N_{0} \tilde{B}_{s}}{1-P_{\text {out }}^{(s, 1)}} \int_{0}^{t_{\text {out }}^{s}} \frac{\exp \left[\frac{\tilde{B}_{s}}{x}-\frac{N_{0}}{\mathcal{P}^{(s, 1)} \Omega_{g_{0}}} \exp \left(\frac{\tilde{B}_{s}}{x}\right)+\frac{N_{0}}{\mathcal{P}^{(s, 1)} \Omega_{g_{0}}}\right]}{x^{2-i}\left[\mathrm{P}(p, 1) \Omega_{\alpha_{1}} \exp \left(\frac{\tilde{B}_{s}}{x}\right)+\mathcal{P}^{(s, 1)} \Omega_{g_{0}}-\mathrm{P}^{(p, 1)} \Omega_{\alpha_{1}}\right]} d x \\
& I_{k}(i)=\int_{0}^{t_{\text {out }}^{s}} \frac{\exp \left[\frac{\tilde{B}_{s}}{x}-\frac{N_{0}}{\mathcal{P}^{(s, 1)} \Omega_{g_{0}}} \exp \left(\frac{\tilde{B}_{s}}{x}\right)+\frac{N_{0}}{\mathcal{P}^{(s, 1)} \Omega_{g_{0}}}\right]}{x^{2-i}\left[\mathrm{P}^{(p, 1)} \Omega_{\alpha_{1}} \exp \left(\frac{\tilde{B}_{s}}{x}\right)+\mathcal{P}^{(s, 1)} \Omega_{g_{0}}-\mathrm{P}^{(p, 1)} \Omega_{\alpha_{1}}\right]^{k}} d x
\end{aligned}
$$




\section{APPENDIX A}

PROOF OF LEMMA 1

1) $C D F$ of $Z$ : Following the probability definition, the cdf of $Z$ can be defined as

$$
\begin{aligned}
F_{Z}(z) & =\operatorname{Pr}\left\{d\left[\ln \left(1+\frac{a X_{1}}{b X_{2}+c}\right)\right]^{-1}<z\right\} \\
& =1-\operatorname{Pr}\left\{\frac{a X_{1}}{b X_{2}+c}<\exp \left(\frac{d}{z}\right)-1\right\} .
\end{aligned}
$$

Using the same approach as given in [37, (14)] for (67), we finally obtain the cdf of $Z$ as in (63), shown earlier.

2) PDF of $Z$ : The pdf of $Z$ is obtained by differentiating (63) with respect to $z$ as in (64). With (63) and (64), Lemma 1 is proven.

\section{APPENDIX B}

\section{Proof of LEMMA 2}

The moments of packet transmission time without timeout can be expressed as

$$
\mathbb{E}\left[\left(T_{\text {suc }}^{(s, 1)}\right)^{i}\right]=\int_{0}^{t_{\text {out }}^{s}} x^{i} f_{T_{\text {suc }}^{(s, 1)}}(x) d x, \quad i=1,2 .
$$

Substituting $f_{T_{\mathrm{suc}}^{(s, 1)}}(x)$ given in (36) into (68), we have (65).

For brevity, let us define $I_{k}(i)$ as in (66), shown earlier. Changing variable $t=\exp \left(\tilde{B}_{s} / x\right)-1$ for (66) yields

$$
I_{k}(i)=\int_{\Phi_{s}}^{\infty} \frac{\exp \left(-\frac{N_{0}}{\mathcal{P}^{(s, 1)} \Omega_{g_{0}}} t\right)}{\tilde{B}_{s}^{1-i}[\ln (t+1)]^{i}\left(\mathrm{P}^{(p, 1)} \Omega_{\alpha_{1}} t+\mathcal{P}^{(s, 1)} \Omega_{g_{0}}\right)^{k}} d t
$$

where $\Phi_{s}=\exp \left(\tilde{B}_{s} / t_{\text {out }}^{s}\right)-1$. Finally, an expression for $\mathbb{E}\left[\left(T_{\text {suc }}^{(s, 1)}\right)^{i}\right]$ given in (68) is obtained as

$\mathbb{E}\left[\left(T_{\text {suc }}^{(s, 1)}\right)^{i}\right]=\frac{N_{0} \tilde{B}_{s} I_{1}(i)}{1-P_{\text {out }}^{(s, 1)}}+\frac{\mathcal{P}^{(s, 1)} \mathrm{P}^{(p, 1)} \tilde{B}_{s} \Omega_{g_{0}} \Omega_{\alpha_{1}} I_{2}(i)}{1-P_{\text {out }}^{(s, 1)}}$.

Lemma 2 is proved.

\section{APPENDIX C}

\section{PROOF OF LEMMA 3}

Let us first consider the series given by [38, Eq. (0.112)], i.e.,

$$
\sum_{n=1}^{M} x^{n}=x \frac{1-x^{M}}{1-x}, x<1, M \geq 1 .
$$

Differentiating (71) with respect to $x$ yields

$$
\sum_{n=1}^{M} n x^{n-1}=-\frac{M x^{M}}{1-x}+\frac{1-x^{M}}{1-x}+\frac{x\left(1-x^{M}\right)}{(1-x)^{2}} .
$$

Multiplying both sides of (72) with $(1-x)$ and let $M \rightarrow \infty$, we reach

$$
\sum_{n=1}^{\infty} n x^{n-1}(1-x)=\frac{1}{1-x} .
$$

Second, we differentiate (72) with respect to $x$ as

$$
\begin{array}{r}
\sum_{n=1}^{M} n(n-1) x^{n-2}=\frac{-M x^{M-1}(1+M)}{1-x}-\frac{2 M x^{M}}{(1-x)^{2}} \\
+\frac{2\left(1-x^{M}\right)}{(1-x)^{2}}+\frac{2 x\left(1-x^{M}\right)}{(1-x)^{3}} .
\end{array}
$$

Multiplying both sides of (74) with $x(1-x)$ yields

$$
\begin{gathered}
\sum_{n=1}^{M} n^{2} x^{n-1}(1-x)=\sum_{n=1}^{M} n x^{n-1}(1-x)-M x^{M}(1+M) \\
-\frac{2 M x^{M+1}}{1-x}+\frac{2 x\left(1-x^{M}\right)}{1-x}+\frac{2 x^{2}\left(1-x^{M}\right)}{(1-x)^{2}} .
\end{gathered}
$$

Now, let $M \rightarrow \infty$ in (75), which yields

$$
\sum_{n=1}^{\infty} n^{2} x^{n-1}(1-x)=\frac{1+x}{(1-x)^{2}} .
$$

Finally, by substituting $x=P_{\text {out }}^{(s, 1)}$ into (73) and (76), Lemma 3 is proved.

\section{APPENDiX D}

PROOF OF LEMMA 4

From (43), the first moment of the interarrival time $A=$ $T_{\text {suc }}^{(s, 1)}+\left(N^{(s, 1)}-1\right) t_{\text {out }}^{s}$ can be expressed as

$$
\mathbb{E}[A]=\mathbb{E}\left[T_{\text {suc }}^{(s, 1)}\right]-t_{\text {out }}^{s}+t_{\text {out }}^{s} \mathbb{E}\left[N^{(s, 1)}\right]
$$

where $\mathbb{E}\left[T_{\text {suc }}^{(s, 1)}\right]$ and $\mathbb{E}\left[N^{(s, 1)}\right]$ are given, respectively, in (40) and (45) as

$$
\begin{aligned}
& \mathbb{E}\left[T_{\text {suc }}^{(s, 1)}\right]=\frac{N_{0} \tilde{B}_{s} I_{1}(1)}{1-P_{\text {out }}^{(s, 1)}}+\frac{\mathcal{P}^{(s, 1)} \mathrm{P}^{(p, 1)} \tilde{B}_{s} \Omega_{g_{0}} \Omega_{\alpha_{1}} I_{2}(1)}{1-P_{\text {out }}^{(s, 1)}} \\
& \mathbb{E}\left[N^{(s, 1)}\right]=\frac{1}{1-P_{\text {out }}^{(s, 1)}} .
\end{aligned}
$$

Substituting (78) and (79) into (77), a tractable expression for the first moment of interarrival time is obtained as

$$
\mathbb{E}[A]=\frac{\mathcal{P}^{(s, 1)} \mathrm{P}^{(p, 1)} \tilde{B}_{s} \Omega_{g_{0}} \Omega_{\alpha_{1}} I_{2}(1)}{1-P_{\text {out }}^{(s, 1)}}+\frac{N_{0} \tilde{B}_{s} I_{1}(1)}{1-P_{\text {out }}^{(s, 1)}}+\frac{t_{\text {out }}^{s} P_{\text {out }}^{(s, 1)}}{1-P_{\text {out }}^{(s, 1)}} .
$$

Furthermore, we can also present the variance of interarrival time as

$$
\begin{aligned}
\operatorname{Var}[A]=\mathbb{E}\left[\left(T_{\text {suc }}^{(s, 1)}+\left(N^{(s, 1)}-1\right) t_{\text {out }}^{s}\right)^{2}\right] \\
-\mathbb{E}^{2}\left[T_{\text {suc }}^{(s, 1)}+\left(N^{(s, 1)}-1\right) t_{\text {out }}^{s}\right] .
\end{aligned}
$$


As the RVs $T_{\text {suc }}^{(s, 1)}$ and $N^{(s, 1)}$ are mutual independent, $\operatorname{Var}[A]$ can be rewritten as follows:

$$
\begin{aligned}
\operatorname{Var}[A]= & \mathbb{E}\left[\left(T_{\text {suc }}^{(s, 1)}\right)^{2}\right]-\mathbb{E}^{2}\left[T_{\text {suc }}^{(s, 1)}\right] \\
& +\left(t_{\text {out }}^{s}\right)^{2}\left\{\mathbb{E}\left[\left(N^{(s, 1)}\right)^{2}\right]-\mathbb{E}^{2}\left[N^{(s, 1)}\right]\right\} \\
= & \operatorname{Var}\left[T_{\text {suc }}^{(s, 1)}\right]+\left(t_{\text {out }}^{s}\right)^{2} \operatorname{Var}\left[N^{(s, 1)}\right]
\end{aligned}
$$

where $\operatorname{Var}\left[N^{(s, 1)}\right]$ can be calculated by using the results in Lemma 3 as

$$
\begin{aligned}
\operatorname{Var}\left[N^{(s, 1)}\right] & =\mathbb{E}\left[\left(N^{(s, 1)}\right)^{2}\right]-\left(\mathbb{E}\left[N^{(s, 1)}\right]\right)^{2} \\
& =\frac{P_{\text {out }}^{(s, 1)}}{\left(1-P_{\text {out }}^{(s, 1)}\right)^{2}}
\end{aligned}
$$

As a consequence, substituting (83) into (82) yields the variance of interarrival time as

$$
\operatorname{Var}[A]=\operatorname{Var}\left[T_{\text {suc }}^{(s, 1)}\right]+\frac{\left(t_{\text {out }}^{s}\right)^{2} P_{\text {out }}^{(s, 1)}}{\left(1-P_{\text {out }}^{(s, 1)}\right)^{2}}
$$

Finally, from (80) and (84), Lemma 4 is proved.

\section{REFERENCES}

[1] J. Mitola and G. Q. Maguire, "Cognitive radio: Making software radios more personal," IEEE Pers. Commun., vol. 6, no. 4, pp. 13-18, Aug. 1999.

[2] A. Goldsmith, S. Jafar, I. Maric, and S. Srinivasa, "Breaking spectrum gridlock with cognitive radios: An information theoretic perspective," Proc. IEEE, vol. 97, no. 5, pp. 894-914, May 2009.

[3] H. Tran, "Performance analysis of cognitive radio networks with interference constraints," Ph.D. dissertation, Blekinge Inst. Technol., Karlskrona, Sweden, Mar. 2013.

[4] H. Tran, T. Duong, and H.-J. Zepernick, "Delay performance of cognitive radio networks for point-to-point and point-to-multipoint communications," EURASIP J. Wireless Commun. Netw., vol. 2012, no. 1, p. 9, Jan. 2012.

[5] O. Simeone, Y. Bar-Ness, and U. Spagnolini, "Stable throughput of cognitive radios with and without relaying capability," IEEE Trans. Wireless Commun., vol. 55, no. 12, pp. 2351-2360, Dec. 2007.

[6] A. Sadek, K. Liu, and A. Ephremides, "Cognitive multiple access via cooperation: Protocol design and performance analysis," IEEE Trans. Inf. Theory, vol. 53, no. 10, pp. 3677-3696, Oct. 2007.

[7] J. Gambini, O. Simeone, U. Spagnolini, Y. Bar-Ness, and Y. Kim, "Cognitive radio with secondary packet-by-packet vertical handover," in Proc. IEEE Int. Conf. Commun., Beijing, China, May 2008, pp. 1050-1054.

[8] J. Gambini, O. Simeone, and U. Spagnolini, "Cognitive relaying and opportunistic spectrum sensing in unlicensed multiple access channels," in Proc. IEEE Int. Symp. Spread Spectr. Tech. Appl., Bologna, Italy, Aug. 2008, pp. 371-375.

[9] H. Suraweera, P. Smith, and N. Surobhi, "Exact outage probability of cooperative diversity with opportunistic spectrum access," in Proc. IEEE Int. Conf. Commun., Beijing, China, May 2008, pp. 79-84.

[10] I. Krikidis, J. Laneman, J. Thompson, and S. McLaughlin, "Protocol design and throughput analysis for multi-user cognitive cooperative systems," IEEE Trans. Wireless Commun., vol. 8, no. 9, pp. 4740-4751, Sep. 2009.

[11] Y. Han, S. H. Ting, and A. Pandharipande, "Cooperative spectrum sharing protocol with secondary user selection," IEEE Trans. Wireless Commun., vol. 9, no. 9, pp. 2914-2923, Sep. 2010.

[12] V. Asghari and S. Aïssa, "Cooperative relay communication performance under spectrum-sharing resource requirements," in Proc. IEEE Int. Conf. Commun., Cape Town, South Africa, May 2010, pp. 1-6.

[13] Y. Chen, V. Lau, S. Zhang, and P. Qiu, "Protocol design and delay analysis of half-duplex buffered cognitive relay systems," IEEE Trans. Wireless Commun., vol. 9, no. 3, pp. 898-902, Mar. 2010.
[14] X. Bao, P. Martins, T. Song, and L. Shen, "Stable throughput analysis of multi-user cognitive cooperative systems," in Proc. IEEE Global Telecommun. Conf., Miami, FL, USA, Dec. 2010, pp. 1-5.

[15] H. Tran, T. Q. Duong, and H.-J. Zepernick, "Performance analysis of cognitive relay networks under power constraints of multiple primary users," in Proc. IEEE Global Telecommun. Conf., Houston, TX, USA, Dec. 2011, pp. 1-6.

[16] Y. Han, S. Ting, and A. Pandharipande, "Cooperative spectrum sharing protocol with selective relaying system," IEEE Commun. Lett., vol. 60, no. 1, pp. 62-67, Jan. 2011.

[17] J. Lee, H. Wang, J. Andrews, and D. Hong, "Outage probability of cognitive relay networks with interference constraints," IEEE Trans. Wireless Commun., vol. 10, no. 2, pp. 390-395, Feb. 2011.

[18] C. Zhong, T. Ratnarajah, and K.-K. Wong, "Outage analysis of decodeand-forward cognitive dual-hop systems with the interference constraint in Nakagami- $m$ fading channels," IEEE Trans. Veh. Technol., vol. 60, no. 6, pp. 2875-2879, Jul. 2011.

[19] H. Ding, J. Ge, D. Benevides da Costa, and Z. Jiang, "Asymptotic analysis of cooperative diversity systems with relay selection in a spectrumsharing scenario," IEEE Trans. Veh. Technol., vol. 60, no. 2, pp. 457-472, Feb. 2011.

[20] S. Sagong, J. Lee, and D. Hong, "Capacity of reactive DF scheme in cognitive relay networks," IEEE Trans. Wireless Commun., vol. 10, no. 10, pp. 3133-3138, Oct. 2011.

[21] M. Khabazian and S. Aïssa, "Modeling and performance analysis of cooperative communications in cognitive radio networks," in Proc. IEEE Int. Symp. Pers. Indoor Mobile Radio Commun., Toronto, ON, Canada, Sep. 2011, pp. 598-603.

[22] T. Q. Duong, V. N. Q. Bao, G. C. Alexandropoulos, and H.-J. Zepernick, "Effect of primary networks on the performance of spectrum sharing AF relaying," Electron. Lett., vol. 48, no. 1, pp. 25-27, Jan. 2012.

[23] H. Kim, S. Lim, H. Wang, and D. Hong, "Optimal power allocation and outage analysis for cognitive full duplex relay systems," IEEE Trans. Wireless Commun., vol. 11, no. 10, pp. 3754-3765, Oct. 2012.

[24] C2POWER, Cognitive radio and cooperative strategies for power saving in multi-standard wireless devices. [Online]. Available: http://www. ict-c2power.eu/

[25] P. Pawelczak, K. Nolan, L. Doyle, S. W. Oh, and D. Cabric, "Cognitive radio: Ten years of experimentation and development," IEEE Commun. Mag., vol. 49, no. 3, pp. 90-100, Mar. 2011.

[26] M. Matinmikko et al., "Cognitive radio trial environment: First live authorized access-based spectrum-sharing demonstration," IEEE Veh. Technol. Mag., vol. 8, no. 3, pp. 30-37, Sep. 2013.

[27] L. Lei, Z. Yingkai, S. X. Sherman, L. Chuang, and Z. Zhangdui, "Performance analysis of device-to-device communications with dynamic interference using stochastic Petri nets," IEEE Trans. Wireless Commun., vol. 12, no. 12, pp. 96-104, Dec. 2013.

[28] F. Gábor et al., "Design aspects of network assisted device-to-device communications," IEEE Commun. Mag., vol. 50, no. 3, pp. 170-177, Mar. 2012.

[29] F. Daquan et al., "Device-to-device communications underlaying cellular networks," IEEE Trans. Commun., vol. 61, no. 8, pp. 3541-3551, Aug. 2013.

[30] V. Chandrasekhar, J. G. Andrews, and A. Gatherer, "Femtocell networks: A survey," IEEE Commun. Mag., vol. 46, no. 9, pp. 56-67, Sep. 2008.

[31] V. Chandrasekhar and J. Andrews, "Uplink capacity and interference avoidance for two-tier cellular networks," in Proc. IEEE Global Telecommun. Conf., Washington, DC, USA, Nov. 2007, pp. 3322-3326.

[32] S. Yeh, S. Talwar, S. Lee, and H. Kim, "WiMAX femtocells: A perspective on network architecture, capacity, coverage," IEEE Commun. Mag., vol. 46, no. 9, pp. 58-65, Sep. 2008.

[33] Y. Zou, J. Zhu, B. Zheng, and Y.-D. Yao, "An adaptive cooperation diversity scheme with best-relay selection in cognitive radio networks," IEEE Trans. Signal Process., vol. 58, no. 10, pp. 5438-5445, Oct. 2010.

[34] N. B. Mehta, V. Sharma, and G. Bansal, "Performance analysis of a cooperative system with rateless codes and buffered relays," IEEE Trans. Wireless Commun., vol. 10, no. 4, pp. 1069-1081, Apr. 2011.

[35] M. Kramer and M. Langenbach-Bellz, "Approximate formulae for the delay in the queueing system GI/G/1," in Proc. Int. Teletraffic Congr., Melbourne, N.S.W., Australia, Nov. 1976, pp. 235-248.

[36] S. M. Ross, Introduction to Probability Models, 9th ed. Amsterdam, The Netherlands: Elsevier, 2007.

[37] Y. Chen, H. Huang, and V. K. N. Lau, "Cooperative spectrum access for cognitive radio network employing rateless code," in Proc. IEEE Int. Conf. Commun., Beijing, China, May 2008, pp. 326-331.

[38] I. Gradshteyn and I. Ryzhik, Table of Integrals, Series, Products, 7th ed. Amsterdam, The Netherlands: Elsevier, 2007. 


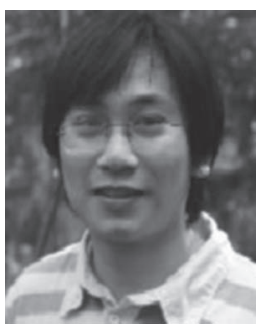

Hung Tran was born in Hanoi, Vietnam, in 1980. $\mathrm{He}$ received the B.S. and M.S. degree in information technology from Vietnam National University, Hanoi, in 2002 and 2006, respectively, and the Ph.D. degree from Blekinge Institute of Technology, Karlskrona, Sweden, in March 2013.

$\mathrm{He}$ is currently a Researcher with the Network Systems Section, Department of Information Technology, National Institute of Education Management, Hanoi. His research interests are in the areas of wireless communications, cognitive radio networks, and green cooperative communication systems.

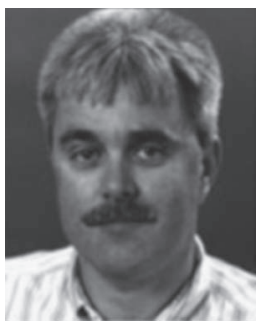

Hans-Jürgen Zepernick (M'94-SM'11) received the Dipl.-Ing. degree from the University of Siegen, Siegen, Germany, in 1987 and the Dr.-Ing. degree from the University of Hagen, Hagen, Germany, in 1994, respectively.

From 1989 to 1989 , he was with Siemens AG, Munich, Germany. He is currently a Professor of radio communications with Blekinge Institute of Technology, Karlskrona, Sweden. Prior to this appointment, he was a Professor of wireless communications with Curtin University of Technology, Bentley, Australia; a Deputy Director with the Australian Telecommunications Research Institute, Perth, Australia; and an Associate Director with the Australian Telecommunications Cooperative Research Centre. His current research interests include cooperative communications, cognitive radio networks, mobile multimedia, and perceptual quality assessment.

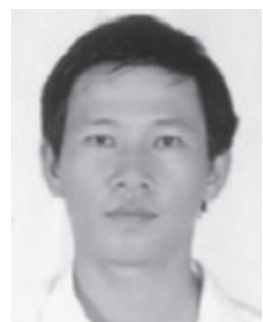

Hoc Phan received the B.S. degree in electronics and computer engineering from Da Nang University of Technology, Da Nang, Vietnam, in 2001; the M.S. degrees in geographic information systems and electrical and electronics engineering from Ho Chi Minh City University of Technology, Ho Chi Minh City, Vietnam, in 2005 and 2006, respectively; and the Ph.D. degree in telecommunication systems from Blekinge Institute of Technology, Karlskrona, Sweden, in March 2013.

In May 2005, he joined the Department of Electrical, Electronics, and Telecommunications, Ho Chi Minh City University of Transport. He is currently a Postdoctoral Research Associate with the University of Reading, Berkshire, U.K. His current research interests include cooperative communications, relay networks, cognitive radio networks, and network coding.

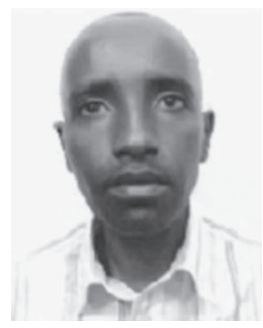

Louis Sibomana received the B.S. and M.S. degrees in telecommunication engineering from the National University of Rwanda, Butare, Rwanda, in 2005 and 2008, respectively. He is currently working toward the Ph.D. degree with the Radio Communications Group, Blekinge Institute of Technology, Karlskrona, Sweden.

From May 2008 to June 2011, he was a Network Performance Analyst and Optimization and Planning Engineer with Rwandatel: a telecommunication company in Rwanda. Since July 2011, he has been an Assistant Lecturer with the Department of Electrical and Electronic Engineering, National University of Rwanda. His research interests include performance analysis of wireless communication systems, cooperative communications, cognitive radio networks, and physical-layer security in wireless networks. 